\title{
A CLIFFORD ALGEBRA APPROACH TO REAL RANK ONE SIMPLE LIE ALGEBRAS
}

\author{
PAOLO CIATTI \\ Dipartimento di Metodi Matematici, Università di Padova \\ Via Belzoni, 7, 35131, Padova, Italy \\ E-mail:ciatti@dmsa.unipd.it
}

Introduction. In this paper we classify and describe real rank one simple Lie algebras $\mathfrak{g}$ with an approach based on the analysis of an Iwasawa nilpotent subalgebra $\mathfrak{n}$ of $\mathfrak{g}$. Our approach is based on the observation of A. Korányi that there is an inner product on $\mathfrak{n}$ which satisfies the compatibility conditions with the Lie product of $\mathfrak{n}$ characteristic of the so called generalized Heisenberg algebras. Korányi, M. Cowling, A. Dooley, and F. Ricci used this fact to classify real rank one simple Lie algebras establishing that the generalized Heisenberg algebras which are Iwasawa satisfy the $J^{2}$-condition, and determining the generalized Heisenberg algebras with this property.

Here is an outline of the paper. In Section 1 after some generalities on real simple Lie algebras and generalized Heisenberg algebras, we give a more detailed account of the contents of the paper. In Section 2 we describe the subalgebra $\mathfrak{m}$ and its action on the root spaces, obtaining a new and easier proof of the $J^{2}$-condition. In Section 3 and Section 4 we provide a uniform construction of real rank one simple Lie algebras starting from a representation of a Clifford algebra. In Section 5 we give some more informations on the structure of $\mathfrak{m}$, proving that $\mathfrak{m}$ coincides with the algebra of skew-symmetric derivations of $\mathfrak{n}$. In Appendix 1 we classify generalized Heisenberg algebras with the $J^{2}$-property using Clifford algebras, and avoiding to use division algebras as done by Korányi and collaborators. Finally, in Appendix 2 we show how our approach can be used to make explicit computations in $\mathfrak{g}$ in the case of $\mathfrak{s p}(1, n)$. The results of Section 2 and Appendix 1 will be also published in Proceedings of the American Mathematical Society [C2].

1. Generalities. Let $\mathfrak{g}$ be a real semi-simple Lie algebra with Killing form $B$. A Car$\tan$ involution $\theta$ of $\mathfrak{g}$ is an involutive automorphism such that the symmetric bilinear form

$$
\langle X, Y\rangle=-c B(X, \theta Y), \quad c>0,
$$

2000 Mathematics Subject Classification: 43A20, 43A90, 53C25.

Received 27 February 2001; revised 6 September 2001. 
is positive definite. Let

$$
\mathfrak{g}=\mathfrak{k} \oplus \mathfrak{p}
$$

be the decomposition of $\mathfrak{g}$ into eigenspaces of $\theta$, where

$$
\mathfrak{k}=\{X \in \mathfrak{g}: \theta X=X\} \quad \text { and } \quad \mathfrak{p}=\{X \in \mathfrak{g}: \theta X=-X\} .
$$

We denote by ad $: \mathfrak{g} \rightarrow$ End $(\mathfrak{g})$ the adjoint representation of $\mathfrak{g}$, defined by

$$
(\operatorname{ad} X) Y=[X, Y], \quad X, Y \in \mathfrak{g} .
$$

If $X$ lies in $\mathfrak{p}$ the operator ad $X$ is symmetric with respect to $\langle\cdot, \cdot\rangle$ and hence diagonalizable. We fix and diagonalize a maximal subalgebra $\mathfrak{a}$ contained in $\mathfrak{p}$ ( $\mathfrak{a}$ is necessarily abelian). The dimension of $\mathfrak{a}$ is an invariant of $\mathfrak{g}$ and is called the real rank of $\mathfrak{g}$. A linear non-zero form $\alpha$ on $\mathfrak{a}$ is called a restricted root, or just a root, relative to the pair $(\mathfrak{g}, \mathfrak{a})$ if the linear space

$$
\mathfrak{g}_{\alpha}=\{X \in \mathfrak{g}:(\operatorname{ad} H) X=\alpha(H) X\}
$$

is non-trivial. In this case $\mathfrak{g}_{\alpha}$ is called the root space of $\alpha$. Denote by $\Sigma$ the set of restricted roots. If $\alpha$ is a root, then $-\alpha$ is also a root and $\mathfrak{g}_{-\alpha}=\theta \mathfrak{g}_{\alpha}$. Moreover, if $\alpha, \beta \in \Sigma$, the linear space

$$
\left[\mathfrak{g}_{\alpha}, \mathfrak{g}_{\beta}\right]=\operatorname{span}\left\{[X, Y]: X \in \mathfrak{g}_{\alpha}, Y \in \mathfrak{g}_{\beta}\right\}
$$

is trivial when $\alpha+\beta \notin \Sigma$, and is a subset of $\mathfrak{g}_{\alpha+\beta}$ when $\alpha+\beta \in \Sigma$. From now on $\mathfrak{g}$ is a simple Lie algebra of real rank one. Hence, $\Sigma$ is either equal to $\{ \pm \alpha\}$, or $\{ \pm \alpha, \pm 2 \alpha\}$. The set $\Sigma$ is called $A_{1}$ in the first case and $B C_{1}$ in the second case. One thus obtains the decomposition

$$
\mathfrak{g}=\theta \mathfrak{n} \oplus \mathfrak{g}_{0} \oplus \mathfrak{n},
$$

where $\mathfrak{n}=\mathfrak{g}_{\alpha}$ for $\Sigma=A_{1}$, and $\mathfrak{n}=\mathfrak{g}_{\alpha} \oplus \mathfrak{g}_{2 \alpha}$ for $\Sigma=B C_{1}$. The subspace $\mathfrak{n}$ is a nilpotent subalgebra of $\mathfrak{g}$.

In this paper we are specially interested in discussing the structure of $\mathfrak{n}$. For this task we shall use the following notion introduced by A. Kaplan in 1980.

1.1. Definition [K]. A nilpotent Lie algebra $\mathfrak{n}=\mathfrak{v} \oplus \mathfrak{z}$ endowed with an inner product $\langle\cdot, \cdot\rangle$, with centre $\mathfrak{z}$ and $\mathfrak{z}^{\perp}=\mathfrak{v}$, is a generalized Heisenberg algebra if the linear map $J_{Z}$ defined for $Z \in \mathfrak{z}$ by

$$
\left\langle J_{Z} X, Y\right\rangle=\langle Z,[X, Y]\rangle \quad \text { for all } X, Y \in \mathfrak{v},
$$

satisfies

$$
J_{Z}^{2}=J_{Z} \circ J_{Z}=-\|Z\|^{2} I \quad \text { for all } Z \in \mathfrak{z} .
$$

From (1.3) it follows by polarization that for $Z, Z^{\prime} \in \mathfrak{z}$

$$
J_{Z} J_{Z^{\prime}}+J_{Z^{\prime}} J_{Z}=-2\left\langle Z, Z^{\prime}\right\rangle I .
$$

Thus, $J: \mathfrak{z} \rightarrow$ End $(\mathfrak{v})$ extends to a representation of the Clifford algebra $\mathcal{C}\left(0, d_{2 \alpha}\right)$ (see for instance $[\mathrm{P}])$. From the definition it also follows easily that $([\mathrm{K}])$

$$
\left[X, J_{Z} X\right]=\|X\|^{2} Z \quad \text { for } X \in \mathfrak{v} \text { and } Z \in \mathfrak{z} .
$$

The group of linear orthogonal automorphisms of a generalized Heisenberg algebra has been extensively studied by C. Riehm $([\mathrm{R}]$, see also $[\mathrm{S}])$. The object of interest now is 
the Lie algebra of this group, that is, the space $\mathcal{D}(\mathfrak{n})$ of the skew-symmetric derivations of $\mathfrak{n}$. If $D \in \mathcal{D}(\mathfrak{n})$, then $D$, being a derivation, maps $\mathfrak{z}$ into itself, and since $D$ is skewsymmetric it also maps $\mathfrak{v}$ into itself. The following result was partially proved in [C, Proposition 3.3] (see also [S]).

1.1. Proposition. (1) A skew-symmetric endomorphism $D$ of the linear space $\mathfrak{n}$ mapping $\mathfrak{z}$ into itself is a derivation of $\mathfrak{n}$ if and only if

$$
D J_{Z}-J_{Z} D=J_{D Z} \text { for all } Z \text { in } \mathfrak{z} \text {. }
$$

In particular, the subalgebra $\mathcal{D}_{0}$ of $\mathcal{D}(\mathfrak{n})$ consisting of the derivations which are trivial on $\mathfrak{z}$ can be identified with the algebra of skew-symmetric linear endomorphisms of $\mathfrak{v}$ wich commute with the action of $\mathcal{C}\left(0, d_{2 \alpha}\right)$.

(2) The linear endomorphism $D_{Z Z^{\prime}}$ of $\mathfrak{n}$ defined for each pair $\left(Z, Z^{\prime}\right)$ of orthogonal vectors of $\mathfrak{z}$ by

$$
\begin{aligned}
D_{Z Z^{\prime}} X & =J_{Z} J_{Z^{\prime}} X \quad \text { for } X \in \mathfrak{v} \quad \text { and } \\
D_{Z Z^{\prime}} Z^{\prime \prime} & =2\left\langle Z, Z^{\prime \prime}\right\rangle Z^{\prime}-2\left\langle Z^{\prime}, Z^{\prime \prime}\right\rangle Z \quad \text { for } Z^{\prime \prime} \in \mathfrak{z},
\end{aligned}
$$

is a derivation of $\mathfrak{n}$. The space

$$
\mathcal{D}_{\mathfrak{z}}=\operatorname{span}\left\{D_{Z Z^{\prime}}: Z, Z^{\prime} \in \mathfrak{z},\left\langle Z, Z^{\prime}\right\rangle=0\right\}
$$

is a Lie algebra isomorphic to $\mathfrak{s o}\left(d_{2 \alpha}\right)$, the Lie algebra of all skew-symmetric linear endomorphisms of $\mathbb{R}^{d_{2 \alpha}}(\simeq z)$, and

$$
\mathcal{D}(\mathfrak{n})=\mathcal{D}_{0} \oplus \mathcal{D}_{\mathfrak{z}} .
$$

In particular, the action of $\mathcal{D}_{0}$ commutes with the action of $\mathcal{D}_{\mathfrak{z}}$.

Proof. (1) Let $D \in \mathcal{D}(\mathfrak{n})$. Since $D$ is skew-symmetric

$$
\left\langle D J_{Z} X, Y\right\rangle=-\left\langle J_{Z} X, D Y\right\rangle=-\langle Z,[X, D Y]\rangle
$$

for $Z \in \mathfrak{z}$ and $X, Y \in \mathfrak{v}$. This relation yields, recalling that $D$ is a derivation and using the definition of $J$,

$$
\begin{aligned}
\left\langle D J_{Z} X, Y\right\rangle & =-\langle Z, D[X, Y]\rangle+\langle Z,[D X, Y]\rangle \\
& =\langle D Z,[X, Y]\rangle+\left\langle J_{Z} D X, Y\right\rangle \\
& =\left\langle J_{D Z} X, Y\right\rangle+\left\langle J_{Z} D X, Y\right\rangle,
\end{aligned}
$$

showing (1.6). These computations read in the opposite direction also prove the converse. The rest of (1) is clear.

(2) By (1.6) $D_{Z Z^{\prime}}$ is a derivation. Notice that $\mathfrak{s o}\left(d_{2 \alpha}\right)$ is isomorphic to $\mathcal{D}_{\mathfrak{z}}$. If $D$ belongs to $\mathcal{D}(\mathfrak{n}),\left.D\right|_{\mathfrak{z}}$ is a skew-symmetric linear transformation of $\mathfrak{z}$, and therefore is the restriction to $\mathfrak{z}$ of an element $D^{\prime}$ of $\mathcal{D}_{\mathfrak{z}}$. Since $D-D^{\prime} \in \mathcal{D}_{0}$ and $\mathcal{D}_{\mathfrak{z}} \cap \mathcal{D}_{0}=\{0\}$, the assertion follows.

The first application of the generalized Heisenberg algebras in the study of real semisimple Lie algebras is due to A. Korányi [Ko], who in 1985 noticed that if $c$ in (1.1) is chosen in such a way that $(\alpha \mid \alpha)=1 / 2$, setting

$$
J_{Z} X=[Z, \theta X], \quad Z \in \mathfrak{g}_{2 \alpha}, X \in \mathfrak{g}_{\alpha},
$$


$J_{Z}$ satisfies $(1.3)$, and therefore $(\mathfrak{n},\langle\cdot, \cdot\rangle)$ is a generalized Heisenberg algebra. After that, he discovered in collaboration with M. Cowling, A. Dooley, and F. Ricci the following property that characterizes in the class of generalized Heisenberg algebras those which derive from the decomposition (1.2) of a real simple Lie algebra (see [CDKR 1], [CDKR $2]$, and $[\mathrm{C} 2])$.

1.2. Definition [CDKR 1]. Let $\mathfrak{n}=\mathfrak{v} \oplus \mathfrak{z}$ be a generalized Heisenberg algebra with centre $\mathfrak{z}$. One says that $\mathfrak{n}$ satisfies the $J^{2}$-condition if for all $X \in \mathfrak{v}$ and all orthogonal pairs $\left(Z, Z^{\prime}\right)$ in $\mathfrak{z}$, there exists $Z^{\prime \prime}$ in $\mathfrak{z}$, (possibly depending on $X, Z$, and $Z^{\prime}$ ), satisfying

$$
J_{Z} J_{Z^{\prime}} X=J_{Z^{\prime \prime}} X \text {. }
$$

This condition is trivially satisfied if $\mathfrak{n}$ is degenerate, i.e. $\mathfrak{z}=\{0\}$, or if $\operatorname{dim} \mathfrak{z}=1$. It is equivalent to requiring that $J_{Z}$ preserves the subspace

$$
\mathbb{R} X \oplus J_{\mathfrak{z}} X=\left\{a X+J_{Z^{\prime}} X: a \in \mathbb{R}, Z^{\prime} \in \mathfrak{z}\right\}
$$

for any $X$ in $\mathfrak{v}$ and any $Z$ in $\mathfrak{z}$, or that the irreducible $\mathcal{C}\left(0, d_{2 \alpha}\right)$-module to which $X$ belongs is equal to $\mathbb{R} X \oplus J_{\mathfrak{z}} X$. From now on we fix $c$ in such a way that $(\alpha \mid \alpha)=1 / 2$. In this paper we present a new proof of the result of Cowling, Dooley, Korányi, and Ricci (Theorem 2.3) and of its main consequence that $d_{2 \alpha}=\operatorname{dim} \mathfrak{g}_{2 \alpha} \in\{0,1,3,7\}$ (Corollary 2.5).

It remains to analyse the subalgebra $\mathfrak{g}_{0}$. It is clear from the definition that

$$
\mathfrak{a}=\mathfrak{g}_{0} \cap \mathfrak{p} .
$$

In our case $\mathfrak{a}$ is one dimensional, and

$$
\mathfrak{a}=\mathbb{R}[V, \theta V] \quad \text { with } V \text { in } \mathfrak{g}_{\alpha} \backslash\{0\} \text { or in } \mathfrak{g}_{2 \alpha} \backslash\{0\} .
$$

In general $\mathfrak{a}$ does not exhaust $\mathfrak{g}_{0}$. We set

$$
\mathfrak{m}=\mathfrak{g}_{0} \cap \mathfrak{k},
$$

so that

$$
\mathfrak{g}_{0}=\mathfrak{a} \oplus \mathfrak{m} .
$$

Then (1.2) gives the Bruhat decomposition of $\mathfrak{g}$

$$
\mathfrak{g}=\theta \mathfrak{n} \oplus \mathfrak{a} \oplus \mathfrak{m} \oplus \mathfrak{n} .
$$

It is easy to see that $\mathfrak{m}$ is a subalgebra and that (see [C1])

$$
\mathfrak{m}=\operatorname{span}\left\{[X, \theta Y]: X, Y \in \mathfrak{g}_{\beta} \text { with } \beta \in\{\alpha, 2 \alpha\} \text { and }\langle X, Y\rangle=0\right\} .
$$

Set also

$$
\mathfrak{m}_{2 \alpha}=\operatorname{span}\left\{\left[Z, \theta Z^{\prime}\right]: Z, Z^{\prime} \in \mathfrak{g}_{2 \alpha} \text { and }\left\langle Z, Z^{\prime}\right\rangle=0\right\}
$$

and

$$
\mathfrak{m}_{\alpha}=\operatorname{span}\left\{[X, \theta Y]: X, Y \in \mathfrak{g}_{\alpha} \text { and }\langle X, Y\rangle=0\right\} .
$$

If $\mathfrak{g}^{(2 \alpha)}$ denotes the subalgebra of $\mathfrak{g}$ generated by the root spaces $\mathfrak{g}_{2 \alpha}$ and $\mathfrak{g}_{-2 \alpha}$, then $\mathfrak{m}_{2 \alpha}=\mathfrak{m} \cap \mathfrak{g}^{(2 \alpha)}$.

The next result holds in a wider context and was proved in [C1] as Corollary 5.2. We present the proof here in an attempt to make the paper self-contained. 
1.2. Proposition. Take a unit vector $X$ in $\mathfrak{g}_{\alpha}$ and two vectors $Z_{1}, Z_{2}$ in $\mathfrak{g}_{2 \alpha}$. Then

$$
\left[\left[Z_{1}, \theta Z_{2}\right], X\right]=J_{1} J_{2} X,
$$

where $J_{1} X$ and $J_{2} X$ stand for $J_{Z_{1}} X$ and $J_{Z_{2}} X$, respectively. Hence, the action of $\mathfrak{m}_{2 \alpha}$ on $\mathfrak{g}_{\alpha}$ coincides with the action of the even subalgebra $\mathcal{C}^{+}\left(d_{2 \alpha}\right)$ of $\mathcal{C}\left(0, d_{2 \alpha}\right)$. Moreover,

$$
\left[Z_{1}, \theta Z_{2}\right]=\left[\theta X, J_{1} J_{2} X\right]+\left[J_{1} X, \theta J_{2} X\right] .
$$

In particular,

$$
\mathfrak{m}_{2 \alpha} \subset \mathfrak{m}_{\alpha} \quad \text { and } \quad \mathfrak{m}=\mathfrak{m}_{\alpha} .
$$

Proof. Formula (1.9) follows from the Jacobi identity and (1.7). Formula (1.10) is also obtained by Jacobi plugging $Z_{2}=\left[X, J_{2} X\right]$ in the left hand side and using (1.7) and (1.9). Finally, from $\left(1.8^{\prime}\right),\left(1.8^{\prime \prime}\right)$, and (1.10) one deduces the last formulæ.

In Proposition 2.1, using only the Jacobi identity and the property of $\mathfrak{m}$ of being fixed by $\theta$, we prove the following formula holding for $X, Y, W \in \mathfrak{g}_{\alpha}$,

$$
\begin{aligned}
{[[X, \theta Y], W] } & =\frac{1}{2}\langle W, X\rangle Y-\frac{1}{2}\langle W, Y\rangle X-\frac{1}{2}\langle X, Y\rangle W \\
& +\frac{1}{2} J_{[X, Y]} W+\frac{1}{2} J_{[X, W]} Y+\frac{1}{2} J_{[W, Y]} X .
\end{aligned}
$$

This formula yields the action of $\mathfrak{m}$ on the linear space $\mathfrak{g}_{\alpha} \oplus \mathfrak{g}_{-\alpha}$, and will be the main tool in our proof of the $J^{2}$-property of $\mathfrak{n}$ (Theorem 2.3).

We denote by $\mathfrak{m}_{2 \alpha}^{\frac{1}{\alpha}}$ the orthogonal complement of $\mathfrak{m}_{2 \alpha}$ in $\mathfrak{m}$, i.e.

$$
\mathfrak{m}_{2 \alpha}^{\perp}=\left\{M \in \mathfrak{m}:\left\langle M, M^{\prime}\right\rangle=0 \text { for all } M^{\prime} \in \mathfrak{m}_{2 \alpha}\right\},
$$

obtaining the decomposition

$$
\mathfrak{m}=\mathfrak{m}_{2 \alpha} \oplus \mathfrak{m}_{2 \alpha}^{\perp} .
$$

1.3. Proposition. The subspaces $\mathfrak{m}_{2 \alpha}$ and $\mathfrak{m}_{2 \alpha}^{\perp}$ are ideals of $\mathfrak{m}$, and

$$
\left[\mathfrak{m}_{2 \alpha}, \mathfrak{m}_{2 \alpha}^{\perp}\right] \subset \mathfrak{m}_{2 \alpha} \cap \mathfrak{m}_{2 \alpha}^{\perp}=0 .
$$

Hence, $\mathfrak{m}_{2 \alpha}^{\perp}$ is the algebra of all skew-symmetric linear endomorphisms of $\mathfrak{g}_{\alpha}$ which commute with the action of $\mathcal{C}^{+}\left(d_{2 \alpha}\right)$.

Proof. It follows from the Jacobi identity that $\mathfrak{m}_{2 \alpha}$ is an ideal in $\mathfrak{m}$. Thus, $\mathfrak{m}_{2 \alpha}^{\perp}$ is also an ideal, and $\mathfrak{m}_{2 \alpha} \cap \mathfrak{m}_{2 \alpha}^{\perp}$ is an ideal in both $\mathfrak{m}_{2 \alpha}$ and $\mathfrak{m}_{2 \alpha}^{\perp}$. Hence, $\mathfrak{m}_{2 \alpha} \cap \mathfrak{m}_{2 \alpha}^{\perp}$ which is trivial, contains $\left[\mathfrak{m}_{2 \alpha}, \mathfrak{m}_{2 \alpha}^{\perp}\right]$. The last part of the assertion follows now by (1.9).

REMARK. We shall see in Corollary 2.6 that $\mathfrak{m}_{2 \alpha}^{\perp}$ is actually the algebra of skewsymmetric linear transformations of $\mathfrak{n}$ commuting with the action of the full algebra $\mathcal{C}\left(0, d_{2 \alpha}\right)$.

We close the section with the following result, holding in a wider context than real rank one simple Lie algebras, which yields another evidence for Clifford algebras in real semi-simple Lie algebras.

1.4. Proposition. Fix an orthonormal basis $\left\{Z_{1}, \ldots, Z_{d_{2 \alpha}}\right\}$ of $\mathfrak{g}_{2 \alpha}$.

(1) The set of endomorphisms of the linear space $\mathfrak{g}_{\alpha} \oplus \mathfrak{g}_{-\alpha}$ defined by $K_{i}=\operatorname{ad} Z_{i}-\operatorname{ad} \theta Z_{i} \quad$ for $i \in\left\{1, \ldots, d_{2 \alpha}\right\}, \quad K_{d_{2 \alpha}+1}=\operatorname{ad}\left[\theta Z_{1}, Z_{1}\right], \quad K_{d_{2 \alpha}+2}=\theta$, 
provides a representation of the Clifford algebra $\mathcal{C}\left(d_{2 \alpha}+2,0\right)$, i.e.

$$
K_{i} K_{j}+K_{j} K_{i}=2 \delta_{i j} I \quad \text { for } i, j \in\left\{1, \ldots, d_{2 \alpha}+2\right\} .
$$

(2) The linear transformations

$$
L_{i}=K_{i} K_{d_{2 \alpha}+1}=K_{i} \operatorname{ad}\left[\theta Z_{1}, Z_{1}\right]=\operatorname{ad} Z_{i}+\operatorname{ad} \theta Z_{i} \quad \text { for } i \in\left\{1, \ldots, d_{2 \alpha}\right\},
$$

yield a representation of the Clifford algebra $\mathcal{C}\left(0, d_{2 \alpha}\right)$ on $\mathfrak{g}_{\alpha} \oplus \mathfrak{g}_{-\alpha}$, i.e.

$$
L_{i} L_{j}+L_{j} L_{i}=-2 \delta_{i j} I \quad \text { for } i, j \in\left\{1, \ldots, d_{2 \alpha}\right\} .
$$

They satisfy for $i, j \in\left\{1, \ldots, d_{2 \alpha}\right\}$ and $i \neq j$

$$
\begin{gathered}
K_{i} L_{i}=-L_{i} K_{i}, \quad K_{i} L_{j}=L_{j} K_{i}, \\
K_{d_{2 \alpha}+1} L_{i}=-L_{i} K_{d_{2 \alpha}+1}, \quad \text { and } \quad K_{d_{2 \alpha}+2} L_{i}=L_{i} K_{d_{2 \alpha}+2} .
\end{gathered}
$$

(3) If $Z \in \mathfrak{g}_{2 \alpha}$ is non-zero,

$$
\mathfrak{g}_{\alpha}=\operatorname{ker}\left(L_{Z}+K_{Z}\right) \quad \text { and } \quad \mathfrak{g}_{-\alpha}=\operatorname{ker}\left(L_{Z}-K_{Z}\right) .
$$

(4) Finally, for $Z \in \mathfrak{g}_{2 \alpha}$,

$$
J_{Z}=\frac{1}{2}\left(K_{Z}+L_{Z}\right) K_{d_{2 \alpha}+2}=\frac{1}{2}\left(K_{Z}+L_{Z}\right) \theta=\frac{1}{2} \theta\left(L_{Z}-K_{Z}\right) .
$$

Proof. We only sketch the proof. Since ad $\left.Z_{i}\right|_{\mathfrak{g}_{\alpha}}=0$ and $\left(\operatorname{ad} \theta Z_{i}\right)^{2}=0$, it follows for $i \in\left\{1, \ldots, d_{2 \alpha}\right\}$ and $X \in \mathfrak{g}_{\alpha}$ that

$$
K_{i}^{2} X=\left[Z_{i}-\theta Z_{i},\left[Z_{i}-\theta Z_{i}, X\right]\right]=-\left[Z_{i}-\theta Z_{i},\left[\theta Z_{i}, X\right]\right]=-\left[Z_{i},\left[\theta Z_{i}, X\right]\right],
$$

which by Jacobi, as $(\alpha \mid \alpha)=1 / 2$, yields

$$
K_{i}^{2} X=-\left[X,\left[\theta Z_{i}, Z_{i}\right]\right]=(2 \alpha \mid \alpha)\left\|Z_{i}\right\|^{2} X=X .
$$

For $Y \in \mathfrak{g}_{-\alpha}$ one proceeds similarly. The rest of (1) is immediate. The assertion in (2) follows from (1) by straightforward calculations, (3) is obvious, and (4) is Korányi's formula (1.7).

REMARK. We see in particular from the above proposition that the action of $\mathfrak{p} \cap \mathfrak{g}^{(2 \alpha)}$ on $\mathfrak{g}_{\alpha} \oplus \mathfrak{g}_{-\alpha}$ extends to a representation of $\mathcal{C}\left(d_{2 \alpha}+1,0\right)$.

The above proposition and Formula (1.11) provide the main instruments in our construction of real rank one simple Lie algebras starting in Section 3. Indeed, in that section we build, according to Proposition 1.4, a Lie algebra $\mathfrak{g}_{\mathfrak{u}}$ of endomorphisms of the linear space $\mathfrak{g}_{\alpha} \oplus \mathfrak{g}_{-\alpha}$ from a representation of $\mathcal{C}\left(d_{2 \alpha}+2,0\right)$. Then we prove that $\mathfrak{g}_{\mathfrak{u}}$ is isomorphic to $\mathfrak{s o}\left(d_{2 \alpha}+1,1\right)$ (Theorem 3.2$)$. The algebra $\mathfrak{g}_{\mathfrak{u}}$ yields $\mathfrak{g}^{(2 \alpha)}$. Finally, using Formula (4) in Proposition 1.4 we introduce a structure of generalized Heisenberg Lie algebra on $\mathfrak{n}=\mathfrak{g}_{\alpha} \oplus \mathfrak{g}_{2 \alpha}$ and $\theta \mathfrak{n}=\mathfrak{g}_{-\alpha} \oplus \mathfrak{g}_{-2 \alpha}$ (Theorem 3.4).

In Section 4, we complete the definition of our algebra introducing the bracket of a vector of $\mathfrak{g}_{\alpha}$ and a vector of $\mathfrak{g}_{-\alpha}$ by means of (1.11). The linear span of these brackets is by definition $\mathfrak{a} \oplus \mathfrak{m}$. In Proposition 4.1 we show that if $\mathfrak{n}$ satisfies the $J^{2}$-condition $\mathfrak{m}$ consists of derivations of $\mathfrak{n}$. From this result the proof that we have obtained a Lie algebra will follow easily (Theorem 4.5). Indeed, Proposition 4.1 is the crucial step in our construction, the actual converse of Theorem 2.3. In fact, one can associate using (1.11) 
a space of linear endomorphisms of $\mathfrak{g}_{-\alpha} \oplus \mathfrak{g}_{\alpha}$ to any generalized Heisenberg algebra $\mathfrak{n}$ with $\mathfrak{v}=\mathfrak{g}_{\alpha}$, but only if $\mathfrak{n}$ satisfies the $J^{2}$-condition these are derivations of $\mathfrak{n}$.

Finally, in Section 5 we discuss the way in which a skew-symmetric derivation of $\mathfrak{n}$, or $\theta \mathfrak{n}$, can be expressed as a linear combination of elements of $\mathfrak{m}$, proving that the space of these derivations coincides with $\mathfrak{m}$. We also determine how $\mathfrak{m}$ depending on $d_{2 \alpha}$ splits into the orthogonal sum of $\mathfrak{m}_{2 \alpha}$ and $\mathfrak{m}_{2 \alpha}^{\perp}$.

2. The action of $\mathfrak{m}$. The following proposition provides a formula describing the action of $\mathfrak{m}$ on $\mathfrak{g}_{\alpha}$.

2.1. Proposition. For $X, Y, W \in \mathfrak{g}_{\alpha}$, one has

$$
\begin{aligned}
{[[X, \theta Y], W] } & =\frac{1}{2}\langle W, X\rangle Y-\frac{1}{2}\langle W, Y\rangle X-\frac{1}{2}\langle X, Y\rangle W \\
& +\frac{1}{2}[[X, Y], \theta W]+\frac{1}{2}[[X, W], \theta Y]+\frac{1}{2}[[W, Y], \theta X] .
\end{aligned}
$$

Proof. If one of $X, Y$, and $W$ is zero (2.1) is trivially true. Assume that $X, Y$, and $W$ are not trivial. By Jacobi one obtains

$$
[[X, \theta Y], W]=[[W, \theta Y], X]+[[X, W], \theta Y] .
$$

Plugging

$$
W=\frac{\langle W, Y\rangle}{\|Y\|^{2}} Y+\left(W-\frac{\langle W, Y\rangle}{\|Y\|^{2}} Y\right),
$$

in the first bracket on the right hand side of (2.2), we decompose $[W, \theta Y]$ into the sum of a term lying in $\mathfrak{a}$ and a term lying in $\mathfrak{m}$ which is therefore fixed by $\theta$. Hence,

$$
[[X, \theta Y], W]=-\frac{1}{2}\langle W, Y\rangle X+\left[\left[\theta W-\frac{\langle W, Y\rangle}{\|Y\|^{2}} \theta Y, Y\right], X\right]+[[X, W], \theta Y] .
$$

From this it follows that

$$
[[X, \theta Y], W]=-\langle W, Y\rangle X+[[\theta W, Y], X]+[[X, W], \theta Y],
$$

and, using again the Jacobi identity,

$$
[[X, \theta Y], W]=-\langle W, Y\rangle X+[[\theta W, X], Y]+[[X, Y], \theta W]+[[X, W], \theta Y] .
$$

Now plug

$$
W=\frac{\langle W, X\rangle}{\|X\|^{2}} X+\left(W-\frac{\langle W, X\rangle}{\|X\|^{2}} X\right)
$$

in the first bracket on the right hand side, obtaining

$$
\begin{aligned}
{[[X, \theta Y], W] } & =-\langle W, Y\rangle X+\frac{1}{2}\langle W, X\rangle Y+\left[\left[W-\frac{\langle W, X\rangle}{\|X\|^{2}} X, \theta X\right], Y\right] \\
& +[[X, Y], \theta W]+[[X, W], \theta Y],
\end{aligned}
$$

which yields

$$
[[X, \theta Y], W]=\langle W, X\rangle Y-\langle W, Y\rangle X+[[W, \theta X], Y]+[[X, Y], \theta W]+[[X, W], \theta Y] .
$$


The Jacobi identity gives

$$
\begin{aligned}
{[[X, \theta Y], W] } & =\langle W, X\rangle Y-\langle W, Y\rangle X+[[Y, \theta X], W] \\
& +[[W, Y], \theta X]+[[X, Y], \theta W]+[[X, W], \theta Y] .
\end{aligned}
$$

Plugging in this formula

$$
Y=\frac{\langle Y, X\rangle}{\|X\|^{2}} X+\left(Y-\frac{\langle Y, X\rangle}{\|X\|^{2}} X\right),
$$

one obtains

$$
\begin{aligned}
{[[X, \theta Y], W] } & =\langle W, X\rangle Y-\langle W, Y\rangle X-\frac{1}{2}\langle X, Y\rangle W+\left[\left[\theta Y-\frac{\langle Y, X\rangle}{\|X\|^{2}} \theta X, X\right], W\right] \\
& +[[W, Y], \theta X]+[[X, Y], \theta W]+[[X, W], \theta Y]
\end{aligned}
$$

which implies

$$
\begin{aligned}
{[[X, \theta Y], W] } & =\langle W, X\rangle Y-\langle W, Y\rangle X-\langle X, Y\rangle W-[[X, \theta Y], W] \\
& +[[W, Y], \theta X]+[[X, Y], \theta W]+[[X, W], \theta Y]
\end{aligned}
$$

providing the statement.

When $2 \alpha$ is not a root the last three terms in (2.1) vanish yielding the usual formula which decribes the action of $\mathfrak{s o}\left(d_{\alpha}\right)$ on $\mathbb{R}^{d_{\alpha}}$.

$$
[[X, \theta Y], W]=\frac{1}{2}\langle W, X\rangle Y-\frac{1}{2}\langle W, Y\rangle X+\frac{1}{2}\langle X, Y\rangle W .
$$

When $2 \alpha$ is a root, (2.1) provides by (1.7) Formula (1.11)

$$
\begin{aligned}
{[[X, \theta Y], W] } & =\frac{1}{2}\langle W, X\rangle Y-\frac{1}{2}\langle W, Y\rangle X+\frac{1}{2}\langle X, Y\rangle W \\
& +\frac{1}{2} J_{[X, Y]} W+\frac{1}{2} J_{[X, W]} Y+\frac{1}{2} J_{[W, Y]} X .
\end{aligned}
$$

From (2.3) and (2.4) it follows in particular that $[X, \theta Y]=0$ only if $X=0$ or $Y=0$.

2.2. Lemma. Suppose $\mathfrak{n}=\mathfrak{v} \oplus \mathfrak{z}$ is a generalized Heisenberg algebra satisfying the $J^{2}$-condition. If $Z, Z^{\prime}$ are orthogonal vectors in $\mathfrak{z}$ and $X$ is a unit vector in $\mathfrak{v}$, then

$$
J_{\left[X, J_{Z} J_{Z^{\prime}} X\right]} X=J_{\left[J_{Z} X, J_{Z^{\prime}} X\right]} X=J_{Z} J_{Z^{\prime}} X \text {. }
$$

Proof. If one of $Z$ and $Z^{\prime}$ is zero (2.5) is trivial. Assume $\|Z\|=\left\|Z^{\prime}\right\|=1$ and let $Z^{\prime \prime} \in \mathfrak{z}$ satisfy $J_{Z} J_{Z^{\prime}} X=J_{Z^{\prime \prime}} X$. Then,

$$
J_{\left[X, J_{Z} J_{Z^{\prime}} X\right]} X=J_{\left[X, J_{Z^{\prime \prime}} X\right]} X=J_{Z^{\prime \prime}} X=J_{Z} J_{Z^{\prime}} X,
$$

by (1.5), proving the first equality. Since $Z^{\prime \prime}$ is orthogonal to $Z$ we find

$$
J_{Z^{\prime}} X=-J_{Z}^{2} J_{Z^{\prime}} X=-J_{Z} J_{Z^{\prime \prime}} X=J_{Z^{\prime \prime}} J_{Z} X,
$$

from which by (1.5) it follows that

$$
J_{\left[J_{Z} X, J_{Z^{\prime}} X\right]} X=J_{\left[J_{Z} X, J_{Z^{\prime \prime}} J_{Z} X\right]} X=J_{Z^{\prime \prime}} X=J_{Z} J_{Z^{\prime}} X,
$$

completing the proof. 
The following result was stated and proved in [CDKR 1] (see also [CDKR 2]). Here we recall the easier proof given in [C2] which is based through Lemma 2.4 on (2.4) and (1.10).

2.3. Theorem. If a generalized Heisenberg Lie algebra $\mathfrak{n}$ appears in the Bruhat decomposition of a simple real rank one Lie algebra $\mathfrak{g}$, then $\mathfrak{n}$ satisfies the $J^{2}$-condition (see Definition 1.2).

2.4. Lemma. Suppose $\alpha, 2 \alpha \in \Sigma$. Take a unit vector $X$ in $\mathfrak{g}_{\alpha}$ and two orthogonal vectors $Z_{1}, Z_{2}$ in $\mathfrak{g}_{2 \alpha}$. Then

$$
J_{1} J_{2} X=J_{\left[J_{1} X, J_{2} X\right]} X=J_{\left[X, J_{1} J_{2} X\right]} X .
$$

Proof. We prove the following formula

$$
J_{1} J_{2} X=\frac{1}{3} J_{\left[J_{1} X, J_{2} X\right]} X+\frac{2}{3} J_{\left[X, J_{1} J_{2} X\right]} X,
$$

from which one sees that the $J^{2}$-condition holds in $\mathfrak{g}_{\alpha} \oplus \mathfrak{g}_{2 \alpha}$. Then the assertion follows by Lemma 2.2. From (2.4) and (1.4) one has

$$
\left[\left[X, \theta J_{1} X\right], J_{2} X\right]=\frac{1}{2} J_{1} J_{2} X+\frac{1}{2} J_{2} J_{1} X+\frac{1}{2} J_{\left[J_{2} X, J_{1} X\right]} X=\frac{1}{2} J_{\left[J_{2} X, J_{1} X\right]} X .
$$

On the other hand, by Jacobi and (1.5) one obtains

$$
\begin{aligned}
{\left[\left[X, \theta J_{1} X\right], J_{2} X\right] } & =\left[\left[J_{2} X, \theta J_{1} X\right], X\right]+\left[\left[X, J_{2} X\right], \theta J_{1} X\right] \\
& =\left[\left[J_{2} X, \theta J_{1} X\right], X\right]+J_{2} J_{1} X .
\end{aligned}
$$

Using (1.10) replace in the last formula $\left[J_{2} X, \theta J_{1} X\right]$ with $\left[Z_{2}, \theta Z_{1}\right]-\left[X, \theta J_{2} J_{1} X\right]$, obtaining by (1.9)

$$
\begin{aligned}
{\left[\left[X, \theta J_{1} X\right], J_{2} X\right] } & =\left[\left[Z_{2}, \theta Z_{1}\right]-\left[X, \theta J_{2} J_{1} X\right], X\right]+J_{2} J_{1} X \\
& =2 J_{2} J_{1} X-\left[\left[X, \theta J_{2} J_{1} X\right], X\right] .
\end{aligned}
$$

The last term can be computed with (2.4) that provides

$$
\left[\left[X, \theta J_{2} J_{1} X\right], X\right]=\frac{1}{2} J_{2} J_{1} X+J_{\left[X, J_{2} J_{1} X\right]} X,
$$

which plugged in (2.9) yields

$$
\left[\left[X, \theta J_{1} X\right], J_{2} X\right]=\frac{3}{2} J_{2} J_{1} X-J_{\left[X, J_{2} J_{1} X\right]} X .
$$

Now, (2.7) follows by comparison of this relation and (2.8).

2.5. Corollary. $d_{2 \alpha}$ belongs to $\{0,1,3,7\}$.

Proof. The statement follows from the classification of generalized Heisenberg algebras satisfying the $J^{2}$-condition (Theorem 1.1 in [CDKR 1]), or alternatively from Proposition A.1.1 in Appendix A.1.

2.6. COROLLARY. $\mathfrak{m}_{2 \alpha}^{\perp}$ is the algebra of all linear endomorphisms which commute with the action of $\mathcal{C}(0, d)$ on $\mathfrak{g}_{\alpha}$.

Proof. The assertion follows from Proposition 1.3 and Formula (2.6). 
2.7. Proposition. Suppose $d_{2 \alpha}=3$. Let $\left\{Z_{1}, Z_{2}, Z_{3}\right\}$ be an orthonormal basis of $\mathfrak{g}_{2 \alpha}$ and set $\epsilon=J_{Z_{1}} J_{Z_{2}} J_{Z_{3}}$. Then either

$$
\epsilon=I, \quad \text { or } \quad \epsilon=-I,
$$

i.e. the irreducible $\mathcal{C}(0,3)$-modules in which $\mathfrak{g}_{\alpha}$ splits are isotypic.

Proof. Suppose that $X, Y \in \mathfrak{g}_{\alpha}$ satisfy

$$
\epsilon X=X \text { and } \epsilon Y=-Y \text {. }
$$

Assume to fix ideas $X \neq 0$. Since ${ }^{t} \epsilon=\epsilon$ and $\epsilon J_{Z}=J_{Z} \epsilon$ for all $Z$ in $\mathfrak{z}+$ it follows that

$$
\langle X, Y\rangle=\left\langle J_{Z} X, Y\right\rangle=0 \quad \text { for all } Z \in \mathfrak{g}_{2 \alpha},
$$

which implies by Jacobi that ad $[X, \theta Y]$ is trivial on $\mathfrak{g}_{2 \alpha}$, and thus

$$
J_{Z} \circ \operatorname{ad}[X, \theta Y]=\operatorname{ad}[X, \theta Y] \circ J_{Z} \quad \text { for all } Z \in \mathfrak{g}_{2 \alpha} .
$$

Hence,

$$
\epsilon \circ \operatorname{ad}[X, \theta Y]=\operatorname{ad}[X, \theta Y] \circ \epsilon \text {. }
$$

Therefore,

$$
\epsilon[[X, \theta Y], X]=\frac{1}{2} \epsilon Y=-\frac{1}{2} Y
$$

is equal to

$$
[[X, \theta Y], \epsilon X]=[[X, \theta Y], X]=\frac{1}{2} Y,
$$

which yields $Y=0$ and provides the statement.

2.8. Proposition. If $d_{2 \alpha}=7$, then $d_{\alpha}=8$.

Proof. When $d_{\alpha}=8, \mathfrak{m}=\mathfrak{m}_{2 \alpha} \simeq \mathfrak{s o}(7)$ since by Corollary $2.6 \mathfrak{m}_{2 \alpha}^{\perp}$ is contained in the commutator of $\mathcal{C}(7) \simeq \mathbb{R}(8) \oplus \mathbb{R}(8)$. If $d_{\alpha}=8 k$, with $k>1$, there are two non-trivial vectors $X, Y \in \mathfrak{g}_{\alpha}$ satisfying

$$
\left\langle J_{Z} X, Y\right\rangle=\left\langle J_{Z} J_{Z^{\prime}} X, Y\right\rangle=0 \text { for all } Z, Z^{\prime} \in \mathfrak{g}_{2 \alpha} .
$$

This gives

$$
\left[J_{Z} X, Y\right]=0 \quad \text { for all } Z \in \mathfrak{g}_{2 \alpha},
$$

which implies by (1.7) and Jacobi

$$
[[\theta X, Y], Z]=0 \quad \text { for all } Z \in \mathfrak{g}_{2 \alpha} .
$$

This yields a contradiction since $[X, \theta Y]$ lies in $\mathfrak{m}_{2 \alpha}$ and the action of $\mathfrak{m}_{2 \alpha}$ on $\mathfrak{g}_{2 \alpha}$ is faithful by (2.1).

For future reference in the next theorem we summarize some of the results of this section.

2.9. TheOREM. The possible values of $\left(d_{2 \alpha}, d_{\alpha}\right)$ are: $(0, n),(1,2 n),(3,4 n)$, and $(7,8)$, with $n \in \mathbb{N}$.

We recall that by $(1.13) \mathfrak{m}=\mathfrak{m}_{2 \alpha} \oplus \mathfrak{m}_{2 \alpha}^{\perp}$. 
2.10. THEOREM. With the notations of Theorem 2.9,

$$
\begin{aligned}
& \mathfrak{m}_{2 \alpha}=\{0\}, \quad \mathfrak{m}_{2 \alpha}^{\perp} \simeq \mathfrak{s o}(n) \quad \text { for } d_{2 \alpha}=0, \\
& \mathfrak{m}_{2 \alpha} \simeq \mathfrak{s o}(2) \simeq \mathfrak{u}(1), \quad \mathfrak{m}_{2 \alpha}^{\perp} \simeq \mathfrak{s u}(n) \quad \text { for } d_{2 \alpha}=1, \\
& \mathfrak{m}_{2 \alpha} \simeq \mathfrak{s o}(3) \simeq \mathfrak{s p}(1), \quad \mathfrak{m}_{2 \alpha}^{\perp} \simeq \mathfrak{s p}(n) \quad \text { for } d_{2 \alpha}=3, \\
& \mathfrak{m}_{2 \alpha} \simeq \mathfrak{s o}(7), \quad \mathfrak{m}_{2 \alpha}^{\perp}=\{0\} \quad \text { for } d_{2 \alpha}=7 \text {. }
\end{aligned}
$$

Proof. The assertion is immediate for $d_{2 \alpha}=0$, and it is a corollary of the proof of Proposition 2.8 for $d_{2 \alpha}=7$. For the other cases, by Corollary 2.6 the action of $\mathfrak{m}_{2 \alpha}^{\perp}$ commutes with the action of $\mathcal{C}\left(0, d_{2 \alpha}\right)$. When $d_{2 \alpha}=d \in\{1,3\}, \mathfrak{m}_{2 \alpha}^{\perp}$ is the algebra of all skew-symmetric linear endomorphisms of $\mathfrak{g}_{\alpha}$ commuting with the complex (for $d=1$ ), or quaternionic (for $d=3$ ) structure $\left\{J_{1}, \ldots, J_{d}\right\}$. The assertion follows from the definitions of $\mathfrak{s u}(n)$ and $\mathfrak{s p}(n)$.

3. Clifford structures in real simple Lie algebras. In this section we assume that $\mathfrak{g}_{2 \alpha}$ is non-trivial. The construction presented here is inspired by Proposition 1.4, but it is useful in contexts wider than real rank one simple Lie algebras.

Fix a positive integer $d$. Take a module $\mathfrak{w}$ of the Clifford algebra $\mathcal{C}(d+2,0)$ and let $\left\{\gamma_{1}, \ldots, \gamma_{d+2}\right\}$ be a set of linear endomorphisms of $\mathfrak{w}$ satisfying

$$
\gamma_{a} \gamma_{b}+\gamma_{b} \gamma_{a}=2 \delta_{a b} I \text { for } a, b=1, \ldots, d+2,
$$

where $I$ is the identity on $\mathfrak{w}$. There is a euclidean inner product $\langle\cdot, \cdot\rangle$ on $\mathfrak{w}$ with respect to which all the $\gamma_{a}$ 's are orthogonal. With respect to this inner product each $\gamma_{a}$ is symmetric. Let

$$
\theta=\gamma_{d+2}, \quad \sigma=\gamma_{d+1}, \quad Q_{i}=\gamma_{i} \quad \text { and } \quad P_{i}=\gamma_{i} \sigma \quad \text { for } i=1, \ldots, d .
$$

Then $\sigma$ and $\theta$ are anticommuting involutive endomorphisms of $\mathfrak{w}$, i.e.

$$
\sigma^{2}=\theta^{2}=I \text { and } \sigma \theta+\theta \sigma=0 .
$$

Moreover,

$$
P_{i} P_{j}+P_{j} P_{i}=-2 \delta_{i j} \text { and } Q_{i} Q_{j}+Q_{j} Q_{i}=2 \delta_{i j},
$$

that is, $\left\{P_{1}, \ldots, P_{d}\right\}$ provides a representation of the Clifford algebra $\mathcal{C}(0, d)$ and $\left\{Q_{1}, \ldots\right.$, $\left.Q_{d}\right\}$ a representation of the Clifford algebra $\mathcal{C}(d, 0)$. They satisfy the following commutation relations

$$
P_{i} Q_{i}+Q_{i} P_{i}=0 \text { and } P_{i} Q_{j}-Q_{j} P_{i}=0 \quad \text { for } i, j=1, \ldots, d \text { and } i \neq j,
$$

and

$$
\sigma Q_{i}=-Q_{i} \sigma, \quad \theta Q_{i}=-Q_{i} \theta, \quad P_{i} \sigma=-\sigma P_{i}, \quad P_{i} \theta=\theta P_{i} \quad \text { for } i=1, \ldots, d .
$$

Take a $d$-dimensional real linear space $\mathfrak{u}$ endowed with the canonical inner product $\langle\cdot, \cdot\rangle$. Consider the cartesian product $\mathfrak{u} \times\{+,-\}$. Set

$$
\mathfrak{z}_{+}=\mathfrak{u} \times\{+\}, \quad \mathfrak{z}_{-}=\mathfrak{u} \times\{-\},
$$

and write

$$
\begin{gathered}
\mathfrak{u} \times\{+,-\}=\mathfrak{z}_{+} \oplus \mathfrak{z}_{-}, \\
Z^{+}=(Z,+) \quad \text { and } \quad Z^{-}=(Z,-) \quad \text { for } Z \in \mathfrak{u} .
\end{gathered}
$$


Fix an orthonormal basis $\left\{Z_{1}, \ldots, Z_{d}\right\}$ of $\mathfrak{u}$ and define a linear map $\theta_{\mathfrak{z}}$ of $\mathfrak{z}+\oplus \mathfrak{z}$ - onto itself by

$$
\theta_{\mathfrak{z}} Z_{i}^{+}=Z_{i}^{-} \quad \text { and } \quad \theta_{\mathfrak{z}} Z_{i}^{-}=Z_{i}^{+} .
$$

Set also for $Z=\sum_{i=1}^{n} \zeta_{i} Z_{i}^{+} \in \mathfrak{z}_{+}, \zeta_{i} \in \mathbb{R}$,

$$
Q_{Z}=\sum_{i=1}^{n} \zeta_{i} Q_{i} \quad \text { and } \quad P_{Z}=\sum_{i=1}^{n} \zeta_{i} P_{i},
$$

and extend the definition of $Q_{Z}$ and $P_{Z}$ to $\mathfrak{z}_{+} \oplus \mathfrak{z}_{-}$by

$$
P_{\theta_{\mathfrak{z}} Z}=P_{Z} \quad \text { and } \quad Q_{\theta_{\mathfrak{z}} Z}=-Q_{Z} \text {. }
$$

Since $\sigma^{2}=I$ and ${ }^{t} \sigma=\sigma, \sigma$ has two eigenvalues \pm 1 . Let

$$
\mathfrak{w}=\mathfrak{v}_{+} \oplus \mathfrak{v}_{-}
$$

be the decomposition of $\mathfrak{w}$ into the eigenspaces of $\sigma$, where

$$
\mathfrak{v}_{+}=\{X \in \mathfrak{w}: \sigma X=X\} \quad \text { and } \quad \mathfrak{v}_{-}=\{X \in \mathfrak{w}: \sigma X=-X\}=\theta \mathfrak{v}_{+} .
$$

Let $\mathfrak{g}_{\mathfrak{u}}$ be the subalgebra of the Lie algebra of all linear endorphisms of $\mathfrak{w}$ (consisting of the linear space End $(\mathfrak{w})$ equipped with the ordinary commutator $[\cdot, \cdot])$ generated by

$$
\left\{P_{Z}+Q_{Z}, P_{Z}-Q_{Z}: Z \in \mathfrak{u}\right\} .
$$

We shall prove that $\mathfrak{g}_{\mathfrak{u}}$ is isomorphic to $\mathfrak{s o}(d+1,1)$.

3.1. Lemma. For $Z, Z^{\prime} \in \mathfrak{z}^{+}$:

$$
\begin{gathered}
\operatorname{ker}\left(P_{Z}+Q_{Z}\right)=\mathfrak{v}_{+} \quad \text { and } \quad \operatorname{ker}\left(P_{Z}-Q_{Z}\right)=\mathfrak{v}_{-} . \\
\left(P_{Z}+Q_{Z}\right)\left(P_{Z^{\prime}}+Q_{Z^{\prime}}\right)=\left(P_{Z}-Q_{Z}\right)\left(P_{Z^{\prime}}-Q_{Z^{\prime}}\right)=0 . \\
{\left[P_{Z}+Q_{Z}, P_{Z^{\prime}}-Q_{Z^{\prime}}\right]=2\left(Q_{Z} Q_{Z^{\prime}}-Q_{Z^{\prime}} Q_{Z}\right)-2\left(Q_{Z} P_{Z^{\prime}}+Q_{Z^{\prime}} P_{Z}\right) .0 .} \\
Q_{Z} Q_{Z^{\prime}}\left(P_{Z^{\prime}}+Q_{Z^{\prime}}\right)=P_{Z}+Q_{Z} \quad \text { and } \quad Q_{Z} Q_{Z^{\prime}}\left(P_{Z^{\prime}}-Q_{Z^{\prime}}\right)=P_{Z}-Q_{Z} .
\end{gathered}
$$

Proof. (1) is obvious. By linearity it is enough to prove the remaining identities for $Z=Z_{i}^{+}$and $Z^{\prime}=Z_{j}^{+}$. To prove the first in (2) observe that

$$
\left(P_{i}+Q_{i}\right)\left(P_{j}+Q_{j}\right)=Q_{i} Q_{j}(I-\sigma)(I+\sigma)=0
$$

since $\sigma$ anti-commutes with $Q_{i}$ and $\sigma^{2}=I$. These properties of $\sigma$ also yield

$$
\left(P_{i}+Q_{i}\right)\left(P_{j}-Q_{j}\right)=Q_{i} Q_{j}(I-\sigma)^{2}=2 Q_{i}\left(Q_{j}-P_{j}\right)
$$

and

$$
\left(P_{i}-Q_{i}\right)\left(P_{j}+Q_{j}\right)=Q_{i} Q_{j}(I+\sigma)^{2}=2 Q_{i}\left(Q_{j}+P_{j}\right),
$$

which give (3). The first identity in (4) follows from

$$
Q_{i} Q_{j}\left(Q_{j}+P_{j}\right)=Q_{i}(I+\sigma)=Q_{i}+P_{i},
$$

the second one can be proved similarly.

3.2. TheOREM. $\mathfrak{g}_{\mathfrak{u}}$ is isomorphic to $\mathfrak{s o}(1+d, 1)$. The restriction to $\mathfrak{g}_{\mathfrak{u}}$ of the linear map $\Theta$ defined by

$$
\Theta\left(Q_{Z_{1}} \ldots Q_{Z_{q}} P_{Z_{q+1}} \ldots P_{Z_{q+p}}\right)=Q_{\theta_{\mathfrak{z}} Z_{1}} \ldots Q_{\theta_{\mathfrak{z}} Z_{q}} P_{\theta_{\mathfrak{z}} Z_{q+1}} \ldots P_{\theta_{\mathfrak{z}} Z_{q+p}}
$$


is a Cartan involution. The map $\mathfrak{g}_{\mathfrak{u}} \times \mathfrak{w} \rightarrow \mathfrak{w}$ defined by $(S, X) \mapsto S X$ yields an action of $\mathfrak{g}_{\mathfrak{u}}$ on $\mathfrak{w}$.

Proof. By (3.1)

$$
\Theta\left(P_{Z}+Q_{Z}\right)=P_{Z}-Q_{Z} \quad \text { and } \quad \Theta\left(P_{Z}-Q_{Z}\right)=P_{Z}+Q_{Z}
$$

In particular, $\theta_{\mathfrak{u}}$ is involutive.

We show that if $\left(P_{Z}+Q_{Z}\right) X=0$ for all $X \in \mathfrak{w}$, then $Z=0$, and similarly that if $\left(P_{Z}-Q_{Z}\right) X=0$ for all $X \in \mathfrak{w}$, then $Z=0$. We prove the first assertion. For any $X$ in $\mathfrak{v}_{+}$,

$$
0=\left(P_{Z}+Q_{Z}\right) X=Q_{Z}(\sigma+1) X=2 Q_{Z} X
$$

which implies

$$
0=Q_{Z}^{2} X=\|Z\|^{2} X
$$

and hence $Z=0$.

Now by (3) in Lemma 3.1,

$$
\left[P_{i}-Q_{i}, P_{i}+Q_{i}\right]=4 Q_{i} P_{i}=4 \sigma
$$

and for $i \neq j$

$$
\left[P_{i}-Q_{i}, P_{j}+Q_{j}\right]=\left[P_{i}+Q_{i}, P_{j}-Q_{j}\right]=4 Q_{j} Q_{i} .
$$

It follows that $\Theta$ is an automorphism of the Lie algebra $\mathfrak{g}_{\mathfrak{u}}$. From the definition of $\Theta$, being $\sigma=Q_{i} P_{i}$, it follows that $\Theta \sigma=-\sigma$. Perform the diagonalization of $\sigma$. Using Lemma 3.1 one obtains from the above relations

$$
\mathfrak{g}_{\mathfrak{u}}=\mathfrak{g}_{\mathfrak{u}-1} \oplus \mathfrak{g}_{\mathfrak{u} 0} \oplus \mathfrak{g}_{\mathfrak{u} 1}
$$

where

$$
\begin{aligned}
\mathfrak{g}_{\mathfrak{u} 1} & =\operatorname{span}\left\{\frac{1}{2}\left(P_{Z}+Q_{Z}\right): Z \in \mathfrak{z}_{+}\right\} \\
\mathfrak{g}_{\mathfrak{u}-1} & =\operatorname{span}\left\{\frac{1}{2}\left(P_{Z}-Q_{Z}\right): Z \in \mathfrak{z}_{+}\right\}=\Theta \mathfrak{g}_{\mathfrak{u} 1},
\end{aligned}
$$

and

$$
\mathfrak{g}_{\mathfrak{u} 0}=\mathbb{R} \sigma \oplus \mathfrak{m}_{\mathfrak{u}}=\mathbb{R} \sigma \oplus \operatorname{span}\left\{Q_{Z} Q_{Z^{\prime}}: Z, Z^{\prime} \in \mathfrak{z}_{+} \text {and }\left\langle Z, Z^{\prime}\right\rangle=0\right\} .
$$

This proves the first part of the statement. The second half is clear.

We identify the linear span of the sets $\left\{P_{Z}+Q_{Z}: Z \in \mathfrak{u}\right\}$ and $\left\{P_{Z}-Q_{Z}: Z \in \mathfrak{u}\right\}$ respectively with $\mathfrak{z}_{+}$and $\mathfrak{z}_{-}$, writing for $Z, Z^{\prime} \in \mathfrak{z}_{+}$and $W \in \mathfrak{w}$

$$
\begin{aligned}
& (\operatorname{ad} Z) W=[Z, W]=\left(P_{Z}+Q_{Z}\right) W, \quad\left(\operatorname{ad} \theta_{\mathfrak{z}} Z\right) W=\left[\theta_{\mathfrak{z}} Z, W\right]=\left(P_{Z}-Q_{Z}\right) W, \\
& \text { and } \quad \operatorname{ad}\left(\left[Z, \theta_{\mathfrak{z}} Z^{\prime}\right]\right)=\left[P_{Z}+Q_{Z}, \Theta\left(P_{Z^{\prime}}+Q_{Z^{\prime}}\right)\right]=\left[P_{Z}+Q_{Z}, P_{Z^{\prime}}-Q_{Z^{\prime}}\right] .
\end{aligned}
$$

For $Z \in \mathfrak{z}_{+}$, we define, according to Proposition 1.4,

$$
J_{Z}=\frac{1}{2}\left(Q_{Z}-P_{Z}\right) \theta
$$

3.3. Lemma. Let $Z \in \mathfrak{z}_{+}$and $Z^{\prime} \in \mathfrak{z}_{-}$. Then,

$$
\left.J_{Z}\right|_{\mathfrak{v}_{+}}=Q_{Z} \theta,\left.\quad J_{Z}\right|_{\mathfrak{v}_{-}}=0 \quad \text { and }\left.\quad J_{\theta_{\mathfrak{z}} Z^{\prime}}\right|_{\mathfrak{v}_{-}}=-Q_{Z^{\prime}} \theta_{\mathfrak{z}},\left.\quad J_{\theta Z^{\prime}}\right|_{\mathfrak{v}_{+}}=0 .
$$


Moreover,

$$
\sigma J_{Z}=J_{Z} \sigma \quad \text { and } \quad J_{Z} \theta+\theta J_{Z}=0
$$

The operators

$$
J_{Z}^{+}=\left.J_{Z}\right|_{\mathfrak{v}_{+}}, \quad Z \in \mathfrak{z}_{+}, \quad \text { and } \quad J_{Z^{\prime}}^{-}=\left.J_{\theta_{\mathfrak{z}} Z^{\prime}}\right|_{\mathfrak{v}_{-}}, \quad Z^{\prime} \in \mathfrak{z}_{-},
$$

satisfy, for $Z_{1}, Z_{2} \in \mathfrak{z}_{+}$and $Z_{1}^{\prime}, Z_{2}^{\prime} \in \mathfrak{z}_{-}$,

$$
J_{Z_{1}}^{+} J_{Z_{2}}^{+}+J_{Z_{2}}^{+} J_{Z_{1}}^{+}=-2\left\langle Z_{1}, Z_{2}\right\rangle I_{\mathfrak{v}_{+}} \quad \text { and } \quad J_{Z_{1}^{\prime}}^{-} J_{Z_{2}^{\prime}}^{-}+J_{Z_{2}^{\prime}}^{-} J_{Z_{1}^{\prime}}^{-}=-2\left\langle Z_{1}^{\prime}, Z_{2}^{\prime}\right\rangle I_{\mathfrak{v}_{-}} .
$$

Hence, $J^{+}: \mathfrak{z}_{+} \rightarrow \operatorname{End}\left(\mathfrak{v}_{+}\right)$and $J^{-}: \mathfrak{z}_{-} \rightarrow \operatorname{End}\left(\mathfrak{v}_{-}\right)$extend to representations of the Clifford algebra $\mathcal{C}(0, d)$. Finally, $J_{Z}^{+}$and $J_{Z^{\prime}}^{-}$are skew-symmetric with respect to the restrictions of $\langle\cdot, \cdot\rangle$ to $\mathfrak{v}_{+}$and $\mathfrak{v}_{-}$, respectively.

Proof. Formulæ (1) and (2) follow directly from (3.4) and from

$$
J_{\theta_{\mathfrak{z}} Z^{\prime}}=\frac{1}{2}\left(Q_{\theta_{\mathfrak{z}} Z^{\prime}}-P_{\theta_{\mathfrak{z}} Z^{\prime}}\right) \theta=-\frac{1}{2}\left(Q_{Z^{\prime}}+P_{Z^{\prime}}\right) \theta=-\frac{1}{2} Q_{Z^{\prime}} \theta(1-\sigma),
$$

which holds for $Z^{\prime} \in \mathfrak{z}_{-}$(recall that by definition $Q_{\theta_{\mathfrak{z}} Z}=-Q_{Z}$ and $P_{\theta_{\mathfrak{z}} Z}=P_{Z}$ ). The rest of the assertion is now an immediate consequence.

From this lemma the following theorem immediately follows.

3.4. TheOREM. The brackets defined on the linear spaces

$$
\mathfrak{n}_{+}=\mathfrak{v}_{+} \oplus \mathfrak{z}_{+} \quad \text { and } \quad \mathfrak{n}_{-}=\mathfrak{v}_{-} \oplus \mathfrak{z}_{-},
$$

for $Z_{+}, Z_{+}^{\prime} \in \mathfrak{z}_{+}$and $Z_{-}, Z_{-}^{\prime} \in \mathfrak{z}_{-}$, by

$$
\left[Z_{+}, Z_{+}^{\prime}\right]=\left[Z_{-}, Z_{-}^{\prime}\right]=0
$$

and for $X_{+}, Y_{+} \in \mathfrak{v}_{+}$and $X_{-}, Y_{-} \in \mathfrak{v}_{-}$, by

$$
\left\langle Z_{+},\left[X_{+}, Y_{+}\right]\right\rangle=\left\langle J_{Z_{+}}^{+} X_{+}, Y_{+}\right\rangle \quad \text { and } \quad\left\langle Z_{-},\left[X_{-}, Y_{-}\right]\right\rangle=\left\langle J_{Z_{-}}^{-} X_{-}, Y_{-}\right\rangle
$$

for all $Z_{+} \in \mathfrak{z}_{+}$and $Z_{-} \in \mathfrak{z}_{-}$, provide on $\mathfrak{n}_{+}$and $\mathfrak{n}_{-}$a structure of $H$-type Lie algebra.

We have introduced a bracket on the linear spaces $\mathfrak{g}_{\mathfrak{u}}, \mathfrak{n}_{+}$, and $\mathfrak{n}_{-}$. These spaces by construction are Lie algebras. Furthermore, we have defined the brackets of a vector lying in $\mathfrak{g}_{\mathfrak{u}}$ with a vector lying in $\mathfrak{w}$ by (3.3). It remains to define the bracket of a vector in $\mathfrak{v}_{+}$ with a vector in $\mathfrak{v}_{-}=\theta \mathfrak{v}_{+}$. This will be done in the next section.

4. Construction of real rank one simple Lie algebras. Assume according to Theorem 2.3 that $\mathfrak{n}$, which is equal to $\mathfrak{g}_{\alpha}$ for $\Sigma=\{ \pm \alpha\}$ and to $\mathfrak{g}_{\alpha} \oplus \mathfrak{g}_{2 \alpha}$ for $\Sigma=\{ \pm \alpha, \pm 2 \alpha\}$, satisfies the $J^{2}$-condition. Let $d$ denote the multiplicity of $2 \alpha$. By Corollary 2.5 it follows that $d \in\{0,1,3,7\}$, with $d=0$ for $\Sigma=A_{1}$.

Let $X, Y \in \mathfrak{v}_{+}$. We distinguish the cases $\Sigma=A_{1}$ and $\Sigma=B C_{1}$, and define the bracket of $X$ and $\theta Y$ by (2.3) and (2.4). For $\Sigma=A_{1}$, let $\Phi_{X Y} \in$ End $\left(\mathfrak{v}_{+}\right)$be defined by

$$
\Phi_{X Y} W=\frac{1}{2}\langle W, X\rangle Y-\frac{1}{2}\langle W, Y\rangle X-\frac{1}{2}\langle X, Y\rangle W .
$$

For $\Sigma=B C_{1}$, let $\Phi_{X Y} \in \operatorname{End}\left(\mathfrak{v}_{+}\right)$be defined by

$$
\Phi_{X Y} W=\frac{1}{2}\langle W, X\rangle Y-\frac{1}{2}\langle W, Y\rangle X-\frac{1}{2}\langle X, Y\rangle W
$$




$$
+\frac{1}{2} J_{[X, Y]} W+\frac{1}{2} J_{[X, W]} Y+\frac{1}{2} J_{[W, Y]} X .
$$

To extend $\Phi_{X Y}$ to $\mathfrak{w}$ we require that

$$
\Phi_{X Y} \theta=\theta \Phi_{X Y} .
$$

Observe that $\Phi_{X X}$ is proportional to $\sigma$ and that for all orthogonal vectors $X, Y$ in $\mathfrak{v}_{+}$

$$
\Phi_{X Y} \sigma=\sigma \Phi_{X Y} .
$$

By means of the following result we shall extend $\Phi_{X Y}$ to a derivation of the Lie algebras $\mathfrak{n}^{+}$and $\mathfrak{n}^{-}$.

4.1. Proposition. (1) Let $Z \in \mathfrak{g}_{2 \alpha}$ and $X, Y \in \mathfrak{g}_{\alpha}$. Then,

$$
\Phi_{X Y} J_{Z}-J_{Z} \Phi_{X Y}= \begin{cases}0 & \text { if } Y=\lambda X, \text { with } \lambda \in \mathbb{R}, \\ J_{\left[J_{Z} Y, X\right]} & \text { if }\langle X, Y\rangle=0 .\end{cases}
$$

(2) The linear map $\Xi_{X Y}$ defined by

$$
\Xi_{X Y} W= \begin{cases}\Phi_{X Y} W & \text { if } W \in \mathfrak{w}, \\ {\left[J_{W} Y, X\right]} & \text { if } W \in \mathfrak{z}+\end{cases}
$$

is a derivation of the generalized Heisenberg algebra $\mathfrak{n}_{+}$.

(3) Let also $X^{\prime}, Y^{\prime} \in \mathfrak{g}_{\alpha}$. Then,

$$
\begin{gathered}
\left(\Xi_{X Y} \Xi_{X^{\prime} Y^{\prime}}-\Xi_{X^{\prime} Y^{\prime}} \Xi_{X Y}\right) J_{Z}-J_{Z}\left(\Xi_{X Y} \Xi_{X^{\prime} Y^{\prime}}-\Xi_{X^{\prime} Y^{\prime}} \Xi_{X Y}\right) \\
=J_{\left[J_{\left[J_{Z} Y^{\prime}, X^{\prime}\right]} Y, X\right]}-J_{\left[J_{\left[J_{Z} Y, X\right]} Y^{\prime}, X^{\prime}\right]} \cdot
\end{gathered}
$$

REMARK 1. Here and in the sequel we state results holding for $\mathfrak{n}_{+}$and $\mathfrak{n}_{-}$only for $\mathfrak{n}_{+}$.

REMARK 2. This proposition is the actual converse of Theorem 2.3. Indeed, as we shall see, it is the property of $\Xi_{X Y}$ of being a derivation of $\mathfrak{n}_{+}$which guarantees that the Jacobi identity holds. But $\Xi_{X Y}$, which may be defined for any generalized Heisenberg algebra $\mathfrak{n}_{+}$by (4.2), is a derivation of it only if $\mathfrak{n}_{+}$satisfies the $J^{2}$-property (this is essentially the content of Theorem 2.3).

4.2. Lemma. Let $A \in \operatorname{End}\left(\mathfrak{v}_{+}\right)$and $Z$ be a unit vector in $\mathfrak{z}$. Then

$$
\left[A, J_{Z}\right] J_{Z}=-J_{Z}\left[A, J_{Z}\right] .
$$

Proof. Indeed,

$$
\begin{aligned}
{\left[A, J_{Z}\right] J_{Z} } & =A\left(J_{Z}\right)^{2}-J_{Z} A J_{Z}=-A-J_{Z} A J_{Z} \\
& =\left(J_{Z}\right)^{2} A-J_{Z} A J_{Z}=-J_{Z}\left[A, J_{Z}\right] .
\end{aligned}
$$

Proof of Proposition 4.1. We start by proving (1). If $d=0$ the statement is trivial. It is not restrictive to assume that $X, Y$, and $Z$ are normalized. In the course of the proof $\left\{Z_{1}, \ldots, Z_{k}\right\}$ will always denote an orthonormal set of vectors in $\mathfrak{g}_{2 \alpha}$. If $Y=\lambda X$ the assertion follows from (3.4) since $\Phi_{X X}$ is proportional to $\sigma$. Hence, assume that $X$ and $Y$ are orthogonal. We first suppose that $X$ and $Y$ lie in distinct modules of $\mathcal{C}(0, d)$, that implies $\langle X, Y\rangle=\left\langle J_{Z} X, Y\right\rangle=0$ for all $Z \in \mathfrak{g}_{2 \alpha}$. By Proposition 2.7 we assume $d \leq 3$. We have by $(4.2)$

$$
\Phi_{X Y} J_{1} X=\frac{1}{2} J_{1} Y \quad \text { and } \quad J_{1} \Phi_{X Y} X=\frac{1}{2} J_{1} Y
$$


Hence,

$$
\left[\Phi_{X Y}, J_{1}\right] X=\left(\Phi_{X Y} J_{1}-J_{1} \Phi_{X Y}\right) X=0,
$$

which by (4.7) also yields

$$
\left[\Phi_{X Y}, J_{1}\right] J_{1} X=0,
$$

proving in particular (4.5) for $d=1$. If $d \geq 3$, suppose first $W=J_{2} X$. Using Lemma 2.2 to express $J_{1} J_{2} X$ as $J_{\left[X, J_{1} J_{2} X\right]} X$ and (4.8), one finds

$$
\Phi_{X Y} J_{1} J_{2} X=\frac{1}{2} J_{1} J_{2} Y \quad \text { and } \quad J_{1} \Phi_{X Y} J_{2} X=\frac{1}{2} J_{1} J_{2} Y
$$

which, as required, provides

$$
\left[\Phi_{X Y}, J_{1}\right] J_{2} X=0
$$

Similarly, one proves that

$$
\left[\Phi_{X Y}, J_{1}\right] J_{Z} Y=0 \quad \text { for any } Z \in \mathfrak{g}_{2 \alpha} .
$$

Moreover, if $\langle X, W\rangle=\left\langle J_{Z} X, W\right\rangle=\langle Y, W\rangle=\left\langle J_{Z} Y, W\right\rangle=0$ for all $Z \in \mathfrak{g}_{2 \alpha}$, the identity $\left[\Phi_{X Y}, J_{1}\right] W=0$ is trivial

Suppose now $Y=J_{1} X$. We shall show that

$$
\left[\Phi_{X J_{1} X}, J_{1}\right]=0,
$$

and that

$$
\left[\Phi_{X J_{1} X}, J_{2}\right]=-J_{3},
$$

where $Z_{3}$ is uniquely determined by

$$
J_{1} J_{2} J_{3} X=X
$$

proving (4.5).

We start by discussing the case in which $W$ is orthogonal to $\mathbb{R} X \oplus J_{\mathfrak{z}} X$. Then

$$
\Phi_{X J_{1} X} J_{Z} W=\frac{1}{2} J_{1} J_{Z} W \quad \text { and } \quad J_{Z} \Phi_{X J_{1} X} W=\frac{1}{2} J_{Z} J_{1} W
$$

Therefore,

$$
\left[\Phi_{X J_{1} X}, J_{Z}\right] W=J_{1} J_{Z} W+\left\langle Z, Z_{1}\right\rangle W,
$$

which implies (4.9) and $\left(4.9^{\prime}\right)$ (for $W$ orthogonal to $\mathbb{R} X \oplus J_{\mathfrak{z}} X$ ).

We prove $(4.9)$ and $\left(4.9^{\prime}\right)$ for $W \in \mathbb{R} X \oplus J_{\mathfrak{z}} X$. For the first, we have, for $W=X$,

$$
\Phi_{X J_{1} X} J_{1} X=\frac{1}{2} J_{1} X \text { and } J_{1} \Phi_{X J_{1} X} X=\frac{1}{2} J_{1} X
$$

which imply $\left[\Phi_{X J_{1} X}, J_{1}\right] X=0$. Then by $(4.7)$ we also obtain $\left[\Phi_{X J_{1} X}, J_{1}\right] J_{1} X=0$. Now consider $W=J_{2} X$,

$$
\Phi_{X J_{1} X} J_{1} J_{2} X=\frac{1}{2} J_{1} J_{1} J_{2} X+\frac{1}{2}\left[X, J_{3} X\right] J_{1} X+\frac{1}{2} J_{\left[J_{1} J_{2} X, J_{1} X\right]} X=\frac{1}{2} J_{2} X,
$$

by (4.10) and (1.7), and

$$
J_{1} \Phi_{X J_{1} X} J_{2} X=\frac{1}{2} J_{1} J_{1} J_{2} X+\frac{1}{2} J_{1} J_{2} J_{1} X+\frac{1}{2} J_{1} J_{\left[J_{2} X, J_{1} X\right]} X=\frac{1}{2} J_{2} X,
$$

where we used (1.5) and $J_{1} J_{\left[J_{2} X, J_{1} X\right]} X=J_{1} J_{\left[J_{2} X, J_{3} J_{2} X\right]} X=J_{1} J_{3} X=J_{2} X$. Hence, $\left[\Phi_{X J_{1} X}, J_{1}\right] J_{2} X=0$, concluding the proof of (4.9). 
Consider now the relation $\left(4.9^{\prime}\right)$. We have, for $W=X$,

$$
\Phi_{X J_{1} X} J_{2} X=\frac{1}{2} J_{1} J_{2} X+\frac{1}{2} J_{2} J_{1} X+\frac{1}{2} J_{\left[J_{2} X, J_{1} X\right]} X=\frac{1}{2} J_{3} X,
$$

by (4.10) and (1.7), and

$$
J_{2} \Phi_{X J_{1} X} X=\frac{1}{2} J_{2} J_{1} X+\frac{1}{2} J_{2} J_{\left[X, J_{1} X\right]} X+\frac{1}{2} J_{2} J_{\left[X, J_{1} X\right]} X=\frac{3}{2} J_{3} X,
$$

which yields

$$
\left[\Phi_{X J_{1} X}, J_{2}\right] X=-J_{3} X
$$

It also follows from (4.7) that

$$
\left[\Phi_{X J_{1} X}, J_{2}\right] J_{2} X=-J_{3} J_{2} X=-J_{1} X .
$$

Then consider $W=J_{3} X$,

$$
\Phi_{X J_{1} X} J_{2} J_{3} X=-\Phi_{X J_{1} X} J_{1} X=\frac{3}{2} X
$$

and

$$
J_{2} \Phi_{X J_{1} X} J_{3} X=\frac{1}{2} J_{2} J_{1} J_{3} X+\frac{1}{2} J_{2} J_{3} J_{1} X+\frac{1}{2} J_{2} J_{\left[J_{3} X, J_{1} X\right]} X=\frac{1}{2} X .
$$

These relations yield

$$
\left[\Phi_{X J_{1} X}, J_{2}\right] J_{3} X=X=-J_{3} J_{3} X
$$

which by (4.7) implies

$$
\left[\Phi_{X J_{1} X}, J_{2}\right] J_{1} X=-\left[\Phi_{X J_{1} X}, J_{2}\right] J_{2} J_{3} X=-J_{3} J_{1} X,
$$

completing the discussion of the case $d \leq 3$.

For $d=7$, we show that

$$
\left[\Phi_{X J_{1} X}, J_{2}\right] J_{4} X=-J_{3} J_{4} X .
$$

Let $Z_{5} \in \mathfrak{g}_{2 \alpha}$ be given by $J_{2} J_{4} X=J_{5} X$, then, using (1.7),

$$
\Phi_{X J_{1} X} J_{2} J_{4} X=\Phi_{X J_{1} X} J_{5} X=\frac{1}{2} J_{1} J_{5} X+\frac{1}{2} J_{5} J_{1} X+\frac{1}{2} J_{\left[J_{5} X, J_{1} X\right]} X=\frac{1}{2} J_{6} X,
$$

where $Z_{6}$ satisfies $J_{5} X=J_{1} J_{6} X$. On the other hand, by Lemma 2.2,

$$
\begin{aligned}
J_{2} \Phi_{X J_{1} X} J_{4} X & =\frac{1}{2} J_{2} J_{1} J_{4} X+\frac{1}{2} J_{2} J_{4} J_{1} X+\frac{1}{2} J_{2} J_{\left[J_{4} X, J_{1} X\right]} X \\
& =\frac{1}{2} J_{2} J_{4} J_{1} X=\frac{1}{2} J_{1} J_{5} X=-\frac{1}{2} J_{6} X .
\end{aligned}
$$

Thus

$$
\begin{aligned}
{\left[\Phi_{X J_{1} X}, J_{2}\right] J_{4} X } & =J_{6} X=-J_{1} J_{5} X=J_{4} J_{1} J_{5} J_{4} X=-J_{4} J_{1} J_{2} X \\
& =J_{4} J_{3} X=-J_{3} J_{4} X,
\end{aligned}
$$

as required. Then (2) follows from (1) by Proposition 1.1.

The proof of (3) is an easy and straightforward computation. By (4.5) it follows that

$$
\begin{aligned}
\Xi_{X Y} \Xi_{X^{\prime} Y^{\prime}} J_{Z} & =\Xi_{X Y}\left(J_{Z} \Xi_{X^{\prime} Y^{\prime}}+J_{\left[J_{Z} Y^{\prime}, X^{\prime}\right]}\right) \\
& =\left(J_{Z} \Xi_{X Y}+J_{\left[J_{Z} Y, X\right]}\right) \Xi_{X^{\prime} Y^{\prime}}+\Xi_{X Y} J_{\left[J_{Z} Y^{\prime}, X^{\prime}\right]} \\
& =J_{Z} \Xi_{X Y} \Xi_{X^{\prime} Y^{\prime}}+J_{\left[J_{Z} Y, X\right]} \Xi_{X^{\prime} Y^{\prime}}+\Xi_{X Y} J_{\left[J_{Z} Y^{\prime}, X^{\prime}\right]},
\end{aligned}
$$


from which, since

$$
\begin{aligned}
J_{\left[J_{Z} Y, X\right]} \Xi_{X^{\prime} Y^{\prime}}+\Xi_{X Y} J_{\left[J_{Z} Y^{\prime}, X^{\prime}\right]} & -J_{\left[J_{Z} Y^{\prime}, X^{\prime}\right]} \Xi_{X Y}-\Xi_{X^{\prime} Y^{\prime}} J_{\left[J_{Z} Y, X\right]} \\
= & J_{\left[J_{\left[J_{Z} Y^{\prime}, X^{\prime}\right]} Y, X\right]}-J_{\left[J_{\left[J_{Z} Y, X\right]} Y^{\prime}, X^{\prime}\right]},
\end{aligned}
$$

one obtains the result.

4.3. Corollary. If $\langle X, Y\rangle=0$,

$$
\Phi_{X Y}\left(Q_{Z} \pm P_{Z}\right)-\left(Q_{Z} \pm P_{Z}\right) \Phi_{X Y}=Q_{\left[J_{Z} Y, X\right]} \pm P_{\left[J_{Z} Y, X\right]} .
$$

Proof. The assertion follows from Proposition 4.1 by (3.4).

Let $\mathfrak{m}$ be the Lie algebra generated in End $(\mathfrak{w})$ by the set

$$
\left\{\Xi_{X Y}: X, Y \in \mathfrak{v}_{+},\langle X, Y\rangle=0\right\} \text {. }
$$

From Proposition 4.1 (3) it follows that $\mathfrak{m}$ consists of skew-symmetric derivations of $\mathfrak{n}_{+}$, or $\mathfrak{n}_{-}$. In the next section we shall prove that $\mathfrak{m}$ is actually the linear span of the set described by (4.11). Define for $\Sigma=A_{1}$ and $\Sigma=B C_{1}$

$$
\operatorname{ad}[X, \theta Y]=-\operatorname{ad}[\theta Y, X]=\Xi_{X Y},
$$

by

$$
\begin{gathered}
{[[X, \theta Y], V]=-[V,[X, \theta Y]]=\Xi_{X Y} V \quad \text { for } V \in \mathfrak{w} \quad \text { and }} \\
{[[X, \theta Y], V]=-[V,[X, \theta Y]]=\Xi_{X Y} V-V \Xi_{X Y} \quad \text { for } V \in \mathfrak{g}_{\mathfrak{u}}+\mathfrak{m} .}
\end{gathered}
$$

Abusing notations we shall denote by $\mathfrak{m}$ also the space spanned by the brackets $[X, \theta Y]$. Recalling (3.3) with these notations Corollary 4.3 may be restated as

$$
[[X, \theta Y], Z]=\left[J_{Z} Y, X\right] \quad \text { and } \quad[[X, \theta Y], \theta Z]=\theta\left[J_{Z} Y, X\right]
$$

the second relation follows from the first by (4.3). If $\langle X, Y\rangle=0$, since $\Xi_{X Y}=-\Xi_{Y X}$,

$$
[X, \theta Y]=[\theta X, Y] \text {. }
$$

We therefore extend $\Theta$ to $\mathfrak{g}_{\mathfrak{u}}+\mathfrak{m}$ setting

$$
\Theta[X, \theta Y]=[X, \theta Y]
$$

Set

$$
\mathfrak{g}=\mathfrak{w} \oplus\left(\mathfrak{g}_{\mathfrak{u}}+\mathfrak{m}\right)=\mathfrak{w} \oplus \mathfrak{g}_{\mathfrak{u}} \oplus \mathfrak{m}^{\perp}
$$

and extend $\theta$ to a linear map, also denoted $\theta$, on $\mathfrak{g}$ putting

$$
\left.\theta\right|_{\mathfrak{g}_{\mathfrak{u}}+\mathfrak{m}}=\Theta
$$

Now $\mathfrak{g}$ is endowed with a skew-symmetric product $[\cdot, \cdot]$. We shall see shortly that $\mathfrak{g}$ with the bracket introduced in this and the previous section is a simple Lie algebra, but first we say something more about $\mathfrak{m}$.

4.4. Proposition. Let $Z_{1}, Z_{2}$ be orthogonal unit vectors in $\mathfrak{z}_{+}$. Then for all unit $X$ in $\mathfrak{v}_{+}$,

$$
\operatorname{ad}\left[Z_{1}, \theta Z_{2}\right]=\Xi_{X J_{1} J_{2} X}+\Xi_{J_{1} X J_{2} X}=-\Xi_{X J_{3} X}+\Xi_{J_{1} X J_{2} X}
$$

where $Z_{3}$ in $\mathfrak{z}+$ satisfies $J_{1} J_{2} J_{3} X=X$. In particular, $\mathfrak{m}_{\mathfrak{u}}$ (defined by (3.2)) is a subalgebra of $\mathfrak{m}$. 
Proof. The proof is an easy and straightforward calculation which makes use of Lemma 2.2.

By (4.13) Formula (1.10) holds in $\mathfrak{g}$.

Let $\mathfrak{m}^{\perp}$ be the orthogonal complement of $\mathfrak{m}_{\mathfrak{u}}$ with respect to the trace (of a linear endomorphism of $\mathfrak{w}$ ) in $\mathfrak{m}$. It is easy to see that $\mathfrak{m}^{\perp}$ is the algebra of the skew-symmetric linear endomorphisms of $\mathfrak{w}$ which commute with the action of $\mathcal{C}(d+2,0)$. In general, $\mathfrak{m}^{\perp}$ is not trivial. In particular, this is the case when $\mathfrak{w}$ is a reducible module of $\mathcal{C}(d+2,0)$. Indeed, let

$$
\mathfrak{w}=\mathfrak{w}_{1} \oplus \ldots \oplus \mathfrak{w}_{n},
$$

where $\mathfrak{w}_{1}, \mathfrak{w}_{2}, \ldots, \mathfrak{w}_{n}$ are irreducible modules of $\mathcal{C}(d+2,0)$. Then for any pair $(a, b)$ of distinct elements of $\{1, \ldots, n\}$, if $X_{a} \in \mathfrak{w}_{a}$ and $X_{b} \in \mathfrak{w}_{b}, \Xi_{X_{a} X_{b}}$ lies in $\mathfrak{m}^{\perp}$ by (4.6) since $\left[X_{a}, J_{Z} X_{b}\right]=0$ for all $Z \in \mathfrak{z}_{+}$. Put

$$
\mathfrak{m}_{0}=\operatorname{span}\left\{\Xi_{X_{a} X_{b}}: a, b \in\{1, \ldots, n\} \text { and } a \neq b\right\} .
$$

It is easy to see that $\mathfrak{m}_{0}$ is isomorphic to $\mathfrak{s o}(n)$, the Lie algebra of all skew-symmetric linear endomorphisms of $\mathbb{R}^{n}$.

4.5. TheOrem. To any pair $(d, k)$ in $\{(0, n),(1,2 n),(3,4 n),(7,8): n \in \mathbb{N}\}$ there corresponds exactly one simple Lie algebra $\mathfrak{g}$ with $\operatorname{dim} \mathfrak{g}_{2 \alpha}=d$ and $\operatorname{dim} \mathfrak{g}_{\alpha}=k$.

Proof. We prove that $\mathfrak{g}$ is a Lie algebra showing that the Jacobi identity is satisfied, i.e. that

$$
\left[\left[V_{1}, V_{2}\right], V_{3}\right]=\left[\left[V_{1}, V_{3}\right], V_{2}\right]+\left[V_{1},\left[V_{2}, V_{3}\right]\right] \text { holds for all } V_{1}, V_{2}, V_{3} \text { in } \mathfrak{g} \text {. }
$$

This is implicit in the discussions made in this and the previous section. In fact, $\mathfrak{g}_{\mathfrak{u}}+\mathfrak{m}$, $\mathfrak{n}_{+}$, and $\mathfrak{n}_{-}$are Lie algebras by Theorem 3.2 and Theorem 3.4. Since $\mathfrak{w}$ is a representation space for the Lie algebra $\mathfrak{g}_{\mathfrak{u}}+\mathfrak{m},(4.16)$ with $V_{1}, V_{2}$ in $\mathfrak{g}_{\mathfrak{u}}+\mathfrak{m}$ and $V_{3}$ in $\mathfrak{w}$ is automatically true. Since by Proposition $4.1 \mathfrak{m}$ is a space of derivations of $\mathfrak{n}_{+}$and $\mathfrak{n}_{-}$, (4.16) holds for $V_{3}$ in $\mathfrak{m}$ and $V_{1}, V_{2}$ in $\mathfrak{v}_{+}$, or $V_{1}, V_{2}$ in $\mathfrak{v}_{-}$. Finally, (4.16) with $V_{1}, V_{3}$ in $\mathfrak{v}_{+}$(or $V_{1}, V_{3}$ in $\left.\mathfrak{v}_{-}\right)$and $V_{2}$ in $\mathfrak{v}_{-}\left(V_{2}\right.$ in $\left.\mathfrak{v}_{+}\right)$, is equivalent to

$$
\Phi_{V_{1} V_{2}} V_{3}+\Phi_{V_{2} V_{3}} V_{1}=0
$$

for $\Sigma=A_{1}$, and to

$$
\Phi_{V_{1} V_{2}} V_{3}+J_{\left[V_{3}, V_{1}\right]} V_{2}+\Phi_{V_{2} V_{3}} V_{1}=0
$$

for $\Sigma=B C_{1}$. Both these identities follow from (4.1) and (4.2) with straightforward computations.

The construction we have outlined uniquely determines $[\cdot, \cdot]$. Therefore, any given simple Lie algebra of real rank one coincides with one of our algebras $(\mathfrak{g},[\cdot, \cdot])$ by Theorem 2.3, Proposition 1.4, and Proposition 2.1.

To prove that $\mathfrak{g}$ is simple, let $\mathfrak{h}$ be a non-trivial ideal in $\mathfrak{g}$. If $\sigma$ lies in $\mathfrak{h}, \mathfrak{g} \subset[\sigma, \mathfrak{g}] \subset \mathfrak{h}$ proving the assertion. If there is a non-trivial $V$ in $\mathfrak{h}$ which lies in one of the subspaces $\mathfrak{z}_{+}, \mathfrak{z}_{-}, \mathfrak{v}_{+}$, or $\mathfrak{v}_{-}$, then $[\theta V, V]$ is proportional to $\sigma$ which thus belongs to $\mathfrak{h}$, and $\mathfrak{h} \subset \mathfrak{g}$ by the previous observation. Otherwise, take a non-trivial $U$ in $\mathfrak{h}$. If $\Sigma=A_{1}$, for any 
non-zero $X$ in $\mathfrak{v}_{+}$there is an integer $k$ such that

$$
V=(\operatorname{ad} X)^{k} U \neq 0 \text { and }(\operatorname{ad} X)^{k+1} U=0 .
$$

Thus, $V$ lies in $\mathfrak{v}_{+}$and $\mathfrak{h}$ implying the assertion. Similarly, if $\Sigma=B C_{1}$, for any non-zero $Z$ in $\mathfrak{z}+$ there is an integer $k$ such that

$$
V=(\operatorname{ad} Z)^{k} U \neq 0 \quad \text { and } \quad(\operatorname{ad} Z)^{k+1} U=0 .
$$

Therefore, $V$ belongs to $\mathfrak{n}_{+}=\mathfrak{v}_{+} \oplus \mathfrak{z}_{+}$. When $V$ lies in $\mathfrak{z}_{+}$, or in $\mathfrak{v}_{+}$, the assertion follows. Otherwise, $V=X+Z^{\prime}$ with $X \in \mathfrak{v}_{+}$and $Z^{\prime} \in \mathfrak{z}_{+}$both non-trivial. We have

$$
\mathfrak{h} \ni[[\theta Z, Z], V]=\|Z\|^{2}\left(2 Z^{\prime}+X\right) \text {. }
$$

Hence, $\|Z\|^{2} Z^{\prime}=[[\theta Z, Z], V]-\|Z\|^{2} V \in \mathfrak{h} \backslash\{0\}$, and the proof continues as above.

In the decomposition of $\mathfrak{g}$ in spaces of restricted roots with respect to $\mathfrak{a}=\mathbb{R} \sigma, \mathfrak{z}+$ and $\mathfrak{z}_{-}$correspond to the root spaces $\mathfrak{g}_{2 \alpha}$ and $\mathfrak{g}_{-2 \alpha}$, respectively, and $\mathfrak{v}_{+}$and $\mathfrak{v}_{-}$to $\mathfrak{g}_{\alpha}$ and $\mathfrak{g}_{-\alpha}$. Moreover, $\mathfrak{g}_{0}=\mathbb{R} \sigma \oplus \mathfrak{m}$.

5. Derivations of $\mathfrak{n}_{+}$. In this section we give some further details on the construction of simple Lie algebras of real rank one with the task of proving that $\mathfrak{m}$ is the algebra of skew-symmetric derivations of $\mathfrak{n}_{+}$and $\mathfrak{n}_{-}$. We shall obtain this result discussing the way in which $\mathfrak{m}$ splits into the direct sum of $\mathfrak{m}_{\mathfrak{u}}$ and $\mathfrak{m}^{\perp}$.

Recall that $d$ is the multiplicity of $\mathfrak{g}_{2 \alpha}$ and that a real Clifford algebra has, up to equivalences, one irreducible module, or two irreducible modules of the same dimension. We shall indicate the real linear space supporting the irreducible modules of $\mathcal{C}(d+2,0)$ with $\mathfrak{w}^{\prime}$.

5.1. $d=0$. Since $\mathcal{C}(2,0) \simeq \mathbb{R}(2)$, the algebra of $2 \times 2$ real matrices, $\mathfrak{w}^{\prime}=\mathbb{R}^{2}$ and $\mathfrak{w}=\mathbb{R}^{2 n}$. Being $d=0, \mathfrak{z}_{+}$and $\mathfrak{z}_{-}$are trivial. Therefore, $\mathfrak{g}_{\mathfrak{u}}$ reduces to $\mathbb{R} \sigma$ and $\mathfrak{m}$ to $\mathfrak{m}^{\perp}$. Furthermore, since the algebra of linear endomorphisms of $\mathfrak{w}^{\prime}$ that commute with the action of $\mathcal{C}(2,0)$ is $\mathbb{R}(\simeq \mathcal{C}(0,0)), \mathfrak{m}^{\perp}=\mathfrak{m}$ coincides with $\mathfrak{m}_{0}$ which, as already noted after (4.15), is isomorphic to $\mathfrak{s o}(n)$. Hence,

$$
\mathfrak{g}=\mathfrak{w} \oplus \mathbb{R} \sigma \oplus \mathfrak{m}_{0} .
$$

To handle the case $d>0$ we keep in mind the following formulæ holding for $X \in \mathfrak{v}_{+}$ and $Z \in \mathfrak{z}^{+}$

$$
\begin{aligned}
J_{Z}^{+} X & =J_{Z} X=\left(P_{Z}+Q_{Z}\right) \theta X=[Z, \theta X] \\
J_{\theta Z}^{-} \theta X & =J_{\theta Z} \theta X=\left(P_{Z}-Q_{Z}\right) \theta X=\theta\left(P_{Z}+Q_{Z}\right) X=\theta[Z, \theta X] .
\end{aligned}
$$

We first consider the case $\mathfrak{w}=\mathfrak{w}^{\prime}$, in which for any non-zero $X$ in $\mathfrak{v}_{+}$

$$
\mathfrak{v}_{+}==\mathbb{R} X \oplus\left\{J_{Z} X: Z \in \mathfrak{z}_{+}\right\} .
$$

5.2. $d=1$. Since $\mathcal{C}(3,0) \simeq \mathbb{C}(2)$, the algebra of $2 \times 2$ complex matrices, $\mathfrak{w}^{\prime}=\mathbb{R}^{4}$. Fix a unit vector $Z$ in $\mathfrak{z}_{+}$, then $\mathfrak{z}_{+}=\mathbb{R} Z$ and $\mathfrak{z}_{-}=\mathbb{R} \theta Z$. Since $\operatorname{dim} \mathfrak{v}_{+}=2$, picking a unit vector $X$ in $\mathfrak{v}_{+}$, one obtains

$$
\mathfrak{v}_{+}=\operatorname{span}\left\{X, J_{Z} X\right\} \quad \text { and } \quad \mathfrak{v}_{-}=\operatorname{span}\left\{\theta X, \theta J_{Z} X\right\} .
$$


The algebra of linear endomorphisms of $\mathfrak{w}^{\prime}$ commuting with the action of $\mathcal{C}(3,0)$ is $\mathbb{C} \simeq \mathcal{C}(0,1)$. A generator of this algebra is given by $\lambda=-\gamma_{1} \gamma_{2} \gamma_{3}$. Hence, $\mathfrak{m}^{\perp}=\mathbb{R} \lambda$. Since $X \in \mathfrak{v}_{+}$from Lemma 3.3 (1) it follows that

$$
\lambda X=Q_{Z} \theta \sigma X=Q_{Z} \theta X=J_{Z} X .
$$

The relations

$$
\operatorname{ad}\left[X, \theta J_{Z} X\right]=-\operatorname{ad}\left[\theta J_{Z} X, X\right]=\frac{3}{2} J_{Z}=\frac{3}{2} \lambda
$$

follow easily from (4.5) and (4.2).

5.3. $d=3$. Since $\mathcal{C}(5,0) \simeq \mathbb{H}(2) \oplus \mathbb{H}(2)$, where $\mathbb{H}(2)$ is the algebra of $2 \times 2$ matrices with quaternionic entries, $\mathfrak{w}^{\prime}=\mathbb{R}^{8}$. Fix an orthonormal basis $\left\{Z_{1}, Z_{2}, Z_{3}\right\}$ of $\mathfrak{z}+$. Then $\left\{\theta Z_{1}, \theta Z_{2}, \theta Z_{3}\right\}$ is an orthonormal basis of $\mathfrak{z}_{-}$. Since $J_{1} J_{2} J_{3}$ is symmetric and $\left(J_{1} J_{2} J_{2}\right)^{2}=$ $I, J_{1} J_{2} J_{3}$ has eigenvalues \pm 1 . By formulæ (2) in Lemma $3.3 J_{1} J_{2} J_{3}$ commutes with $\sigma$ and anti-commutes with $\theta$. Therefore, without loss of generality we can assume that $J_{1} J_{2} J_{3}$ restricted to $\mathfrak{v}_{+}$is the identity and restricted to $\mathfrak{v}_{-}$minus the identity. Pick a unit vector $X$ in $\mathfrak{v}_{+}$. Since $\operatorname{dim} \mathfrak{v}_{+}=4$ one obtains

$$
\mathfrak{v}_{+}=\operatorname{span}\left\{X, J_{1} X, J_{2} X, J_{3} X\right\} \quad \text { and } \quad \mathfrak{v}_{-}=\operatorname{span}\left\{\theta X, \theta J_{1} X, \theta J_{2} X, \theta J_{3} X\right\} .
$$

The algebra $\mathfrak{m}^{\perp}$ of skew-symmetric linear endomorphisms of $\mathfrak{w}^{\prime}$ commuting with $\mathcal{C}(5,0)$ is $\mathbb{H} \simeq \mathcal{C}(0,2)$. Let $\left\{\lambda_{1}, \lambda_{2}\right\} \subset$ End $\left(\mathfrak{w}^{\prime}\right)$ be defined by

$$
\begin{array}{cl}
\lambda_{i} \theta=\theta \lambda_{i}, & \lambda_{i} X=J_{i} X, \quad \text { and } \\
\lambda_{i} J_{k} X=J_{k} J_{i} X & \text { for } i=1,2 \text { and } k=1,2,3 .
\end{array}
$$

Then $\left\{\lambda_{1}, \lambda_{2}\right\}$ is a set of generators of $\mathfrak{m}^{\perp}$ which satisfy

$$
\lambda_{a} \lambda_{b}+\lambda_{b} \lambda_{a}=-\delta_{a b} I \quad \text { and } \quad \lambda_{a} \gamma_{i}=\gamma_{i} \lambda_{a} \quad a, b=1,2 \text { and } i=1, \ldots, 5 .
$$

Set also $\lambda_{3}=\lambda_{1} \lambda_{2}$, then

$$
\lambda_{3} X=\lambda_{1} \lambda_{2} X=\lambda_{1} J_{2} X=J_{2} J_{1} X=J_{3} X,
$$

and $\mathfrak{m}^{\perp}=\operatorname{span}\left\{\lambda_{1}, \lambda_{2}, \lambda_{3}\right\} \simeq \mathcal{C}(0,2) \simeq \mathbb{H}$. From (4.2) (see Appendix A.2) one obtains

$$
\begin{aligned}
\operatorname{ad}\left[X, \theta J_{i} X\right] & =-\operatorname{ad}\left[\theta J_{i} X, X\right] \\
& =\frac{1}{2}\left(\lambda_{i}-\frac{1}{2} \sum_{j, k=1}^{3} \epsilon_{i j k} \operatorname{ad}\left[Z_{j}, \theta Z_{k}\right]\right) \quad i=1,2,3,
\end{aligned}
$$

where $\epsilon_{i j k}$ is defined in Appendix A.2, and, using also (4.13),

$$
\begin{aligned}
\operatorname{ad}\left[J_{i} X, \theta J_{k} X\right] & =-\operatorname{ad}\left[\theta J_{i} X, J_{k} X\right] \\
& =\frac{1}{2}\left(\operatorname{ad}\left[Z_{i}, \theta Z_{k}\right]+\sum_{l=1}^{3} \epsilon_{i k l} \lambda_{l}\right) \quad i, k=1,2,3 .
\end{aligned}
$$

Using the second of the (A.2.2)'s to find $\lambda_{i}$ from $\left(5.6^{\prime}\right)$ and summing the result to (5.6) one obtains

$$
\lambda_{i}=\left[X, \theta J_{i} X\right]+\frac{1}{2} \sum_{j, k=1}^{3} \epsilon_{i j k}\left[J_{j} X, \theta J_{k} X\right] .
$$


5.4. $d=7$. Since $\mathcal{C}(9,0) \simeq \mathbb{R}(16) \oplus \mathbb{R}(16), \mathfrak{w}^{\prime}=\mathbb{R}^{16}$. The algebra $\mathfrak{m}^{\perp}$ of skewsymmetric linear endomorphisms of $\mathfrak{w}^{\prime}$ commuting with the action of $\mathcal{C}(9,0)$ is trivial. Therefore $\mathfrak{m}=\mathfrak{m}_{\mathfrak{u}}$, according to Theorem 2.10. Hence,

$$
\mathfrak{g}=\mathfrak{w}^{\prime} \oplus \mathfrak{g}_{\mathfrak{u}} .
$$

Fix a unit vector $X$ in $\mathfrak{v}_{+}$and a unit vector $Z_{1}$ in $\mathfrak{z}_{+}$. We shall write $\left[X, \theta J_{1} X\right]$ as a linear combination of elements of $\mathfrak{m}_{\mathfrak{u}}$. There is an orthonormal basis $\left\{Z_{1}, \ldots, Z_{7}\right\}$ of $\mathfrak{z}_{+}$ such that

$$
J_{1} J_{2} J_{3} X=J_{1} J_{4} J_{5} X=J_{1} J_{6} J_{7} X=X,
$$

which implies

$$
J_{1} \ldots J_{7} X=-X
$$

Let

$$
M_{1}=\left[X, \theta J_{1} X\right]+\left[J_{2} X, \theta J_{3} X\right] .
$$

From (5.8) one obtains

$$
M_{1}=-\left[X, \theta J_{2} J_{3} X\right]+\left[J_{2} X, \theta J_{3} X\right]
$$

and from (4.13)

$$
M_{1}=\left[Z_{2}, \theta Z_{3}\right]+2\left[X, \theta J_{1} X\right]=-\left[Z_{2}, \theta Z_{3}\right]+2\left[J_{2} X, \theta J_{3} X\right] .
$$

By (5.8) it follows from (4.12) and Proposition 4.1, or alternatively using the Jacobi identity (which holds in $\mathfrak{g}$ by Theorem 4.5 ), that

$$
\left[M_{1}, Z_{i}\right]=0 \text { for } i=1,2,3 \text {, }
$$

and that

$$
\left[M_{1}, Z_{4}\right]=-2 Z_{5}, \quad\left[M_{1}, Z_{5}\right]=2 Z_{4}, \quad\left[M_{1}, Z_{6}\right]=-2 Z_{7}, \quad\left[M_{1}, Z_{7}\right]=2 Z_{6} .
$$

Therefore,

$$
M_{1}=-\left[Z_{4}, \theta Z_{5}\right]-\left[Z_{6}, \theta Z_{7}\right],
$$

from which we deduce by (5.9)

$$
\left[X, \theta J_{1} X\right]=-\frac{1}{2}\left[Z_{2}, \theta Z_{3}\right]-\frac{1}{2}\left[Z_{4}, \theta Z_{5}\right]-\frac{1}{2}\left[Z_{6}, \theta Z_{7}\right],
$$

and also

$$
\left[J_{2} X, \theta J_{3} X\right]=\frac{1}{2}\left[Z_{2}, \theta Z_{3}\right]-\frac{1}{2}\left[Z_{4}, \theta Z_{5}\right]-\frac{1}{2}\left[Z_{6}, \theta Z_{7}\right] .
$$

We now discuss the algebras in which $\mathfrak{w}$ is a reducible module of $\mathcal{C}(d+2,0)$ and $d$ is not zero. According to Theorem 2.10 we assume $d=1,3$. The following result describes the action of $\mathfrak{m}$ on $\mathfrak{w}$.

5.1. Proposition. Consider the decomposition (4.14) of $\mathfrak{w}$ and let $X_{a}$ be a unit vector in $\mathfrak{w}_{a}$. If $a \neq b$,

$$
\left[\left[X_{a}, \theta X_{b}\right], J_{Z} X_{c}\right]=J_{Z}\left[\left[X_{a}, \theta X_{b}\right], X_{c}\right]=\frac{1}{2} \delta_{a c} J_{Z} X_{b}-\frac{1}{2} \delta_{b c} J_{Z} X_{a} .
$$


Proof. The assertion follows from Proposition 4.1 since $\left[X_{a}, J_{Z} X_{b}\right]=0$ for all $Z$ in $\mathfrak{g}_{2 \alpha}$.

In the next theorem we prove that in real rank one simple Lie algebras the subalgebra $\mathfrak{m}$ coincides with the space of skew-symmetric derivations of the generalized Heisenberg algebra $\mathfrak{n}_{+}$, a result which is far from being true in higher rank (see [C1] and [CC]).

5.2. THEOREM. $\mathfrak{m}$ coincides with $\mathcal{D}\left(\mathfrak{n}_{+}\right)=\mathcal{D}_{+}$, the algebra of all skew-symmetric derivations of $\mathfrak{n}_{+}$.

Proof. By Proposition $4.1 \Xi_{X Y}$ is a derivation of $\mathfrak{n}_{+}$for any pair $(X, Y)$ of orthogonal vectors in $\mathfrak{v}_{+}$. Clearly, if $X$ and $Y$ are orthogonal $\Xi_{X Y}$ is skew-symmetric and thus lies in $\mathcal{D}_{+}$.

For the converse, observe that $\mathcal{D}_{\mathfrak{z}_{+}}$, the algebra of skew-symmetric derivations which are non-trivial on $\mathfrak{z}_{+}$, is equal to $\mathfrak{m}_{\mathfrak{u}}$ by Proposition $1.1(2)$. Let $D \in \mathcal{D}_{+}$. By Proposition 1.1 there is a derivation $D^{\prime}$ in $\mathcal{D}_{\mathfrak{z}_{+}}$such that $D-D^{\prime} \in \mathcal{D}_{0}$. Pick a unit vector $X$ in $\mathfrak{v}_{+}$, then

$$
D^{\prime} X=J_{\bar{Z}} X+Y
$$

for some $\bar{Z} \in \mathfrak{z}_{+}$and some $Y \in \mathfrak{v}_{+}$orthogonal to $X$ and $J_{Z} X$ for all $Z \in \mathfrak{z}_{+}$, by (5.2). Clearly, $[X, \theta Y]$ belongs to the subalgebra $\mathfrak{m}_{0}$ defined by (4.15). Moreover,

$$
\left(D^{\prime}-2 \operatorname{ad}[X, \theta Y]\right) X=J_{\bar{Z}} X \quad \text { and } \quad\left(D^{\prime}-2 \operatorname{ad}[X, \theta Y]\right) J_{Z} X=J_{Z} J_{\bar{Z}} X
$$

for $Z \in \mathfrak{z}_{+}$. Hence, denoting by $D^{\prime \prime}$ the restriction of $D^{\prime}-2 \operatorname{ad}[X, \theta Y]$ to $\mathbb{R} X \oplus J_{\mathfrak{z}} X, D^{\prime \prime}$ is a linear map of $\mathbb{R} X \oplus J_{\mathfrak{z}} X$ into itself commuting with the action of $\mathcal{C}(0, d)$. Therefore, $D^{\prime \prime}$ is a linear combination of the $\lambda_{i}$ 's. It follows from (5.3), (5.6), and (5.7) that $D^{\prime \prime}$ lies in $\mathfrak{m}$ (actually, $D^{\prime \prime} \in \mathfrak{m}^{\perp}$ ), proving the statement.

From this theorem and Proposition 4.1 (3) one immediately obtains the following result.

\subsection{Corollary.}

$$
\mathfrak{m}=\operatorname{span}\left\{\Xi_{X Y}: X, Y \in \mathfrak{v}_{+},\langle X, Y\rangle=0\right\}
$$

A.1. Appendix. In this appendix we are concerned with a generalized Heisenberg algebra $\mathfrak{n}=\mathfrak{v} \oplus \mathfrak{z}$ with inner product $\langle\cdot, \cdot\rangle$, centre $\mathfrak{z}$ of dimension $d$, and $\mathfrak{v}=\mathfrak{z}^{\perp}$.

A.1.1. Proposition. If $\mathfrak{n}$ satisfies the $J^{2}$-condition, then $d$ belongs to $\{0,1,3,7\}$.

Proof. Observe that by the $J^{2}$-condition if $X$ is a vector in $\mathfrak{v}$ and $Z_{1}, Z_{2}$ are orthogonal unit vectors in $\mathfrak{z}$ such that

$$
\left\langle J_{Z} X, J_{1} J_{2} X\right\rangle=0 \text { for all } Z \text { in } \mathfrak{z},
$$

then $X=0$. We prove the proposition showing by the following two lemmas that this property (which is trivial for $d=0,1$ ) does not hold if $d$ is not in $\{0,1,3,7\}$.

A.1.2. Lemma. Let $d \in\{2,4,5,11\}$ and let $\left\{Z_{1}, \ldots, Z_{d}\right\}$ be an orthonormal basis of $\mathfrak{z}$. Then there is a unit vector $X \in \mathfrak{v}$ satisfying

$$
\left\langle J_{1} J_{2} X, J_{Z} X\right\rangle=0 \quad \text { for all } Z \in \mathfrak{z} .
$$


Proof. For $d=2$, if $Z=a Z_{1}+b Z_{2}$, we have

$$
\left\langle Z,\left[X, J_{1} J_{2} X\right]\right\rangle=\left\langle J_{Z} X, J_{1} J_{2} X\right\rangle=a\left\langle X, J_{2} X\right\rangle-b\left\langle X, J_{1} X\right\rangle=0 .
$$

For $d=4$, let

$$
\epsilon=J_{1} J_{2} J_{3} J_{4} \text {. }
$$

Then $\epsilon$ is symmetric and $\epsilon^{2}=I$. Therefore, there is a unit $X \in \mathfrak{v}$ such that $\epsilon X= \pm X$. Assume to fix ideas that $\epsilon X=X$. Since $\epsilon$ anticommutes with $J_{Z}$ for any $Z \neq 0$ in $\mathfrak{z}$, we have

$$
\epsilon J_{i} X=-J_{i} X \quad \text { and } \quad \epsilon J_{i} J_{k} X=J_{i} J_{k} X \quad \text { for } i, k \in\{1,2,3,4\},
$$

from which it follows

$$
\left\langle J_{i} J_{k} X, J_{l} X\right\rangle=0
$$

yielding $(A .1 .1)$.

For $d=5$, let

$$
\phi=J_{1} J_{2} J_{3} J_{4} \text { and } \eta=J_{2} J_{4} J_{5} .
$$

Then $\phi$ and $\eta$ are symmetric, commute, and $\phi^{2}=\eta^{2}=I$. Hence, there is a unit vector $X$ in $\mathfrak{v}$ such that $\phi X=\eta X=X$. As in the previous case it follows that

$$
\left\langle J_{1} J_{2} X, J_{i} X\right\rangle=0 \quad \text { for } i \in\{1, \ldots, 4\} .
$$

Moreover, since $\eta J_{1} J_{2} X=-J_{1} J_{2} X$ and $\eta J_{5} X=J_{5} X$, we also have

$$
\left\langle J_{1} J_{2} X, J_{5} X\right\rangle=0 \text {. }
$$

For $d=11$, let

$$
\mu=J_{1} J_{3} J_{5} J_{7} \quad \text { and } \quad \nu=J_{2} J_{4} J_{6} J_{8} .
$$

Note that $\mu$ and $\nu$ are symmetric, commute, and $\mu^{2}=\nu^{2}=I$. Hence, there is a unit vector $X$ in $\mathfrak{v}$ satisfying

$$
\mu X=\nu X=X
$$

Call $L, M$, and $N$ the linear spans of $\left\{Z_{9}, Z_{10}, Z_{11}\right\},\left\{Z_{1}, Z_{3}, Z_{5}, Z_{7}\right\}$, and $\left\{Z_{2}, Z_{4}, Z_{6}\right.$, $Z_{8}$ \}, respectively. We have

$$
\mu J_{1} J_{2} X=-J_{1} J_{2} X \quad \text { and } \quad \nu J_{1} J_{2} X=-J_{1} J_{2} X .
$$

Therefore, since $Z \in L$ yields

$$
\mu J_{Z} X=J_{Z} \mu X=J_{Z} X
$$

one obtains by $(A .1 .2)$

$$
\left\langle J_{Z} X, J_{1} J_{2} X\right\rangle=0 \quad \text { for all } Z \in \mathfrak{z} .
$$

Similarly, since $Z \in M$ implies

$$
\nu J_{Z} X=J_{Z} \nu X=J_{Z} X,
$$

it follows from $(A .1 .2)$ that $J_{Z} X$ is orthogonal to $J_{1} J_{2} X$. Finally, the same argument with $\mu$ in place of $\nu$ shows that $J_{Z} X$ is orthogonal to $J_{1} J_{2} X$ for $Z$ in $N$ yielding (A.1.1).

A.1.3. Lemma. Let $d=m+4, m \geq 2$. If

$$
\left\langle J_{1} J_{2} X, J_{i} X\right\rangle=0 \quad \text { for all } i \in\{1, \ldots, m\},
$$


then

$$
\left\langle J_{1} J_{2} X, J_{i} X\right\rangle=0 \quad \text { for all } i \in\{1, \ldots, m+4\} \text {. }
$$

Proof. Set

$$
\chi=J_{m+1} J_{m+2} J_{m+3} J_{m+4} .
$$

It is clear that $\chi$ is symmetric and that $\chi^{2}=I$. As before there is a unit vector $X \in \mathfrak{v}$ satisfying $\chi X=X$. Since

$$
J_{i} \chi=\chi J_{i} \quad \text { for } i \in\{1, \ldots, m\} \text {, }
$$

it follows that

$$
\chi J_{1} J_{2} X=J_{1} J_{2} X
$$

from which, as

$$
J_{i} \chi=-\chi J_{i} \quad \text { for } i \in\{m+1, \ldots, m+4\} \text {, }
$$

one obtains

$$
\left\langle J_{1} J_{2} X, J_{i} X\right\rangle=0 \quad \text { for } i \in\{1, \ldots, m+4\}
$$

A.2. Appendix. We compute the brackets in $\mathfrak{n}_{+}$for $d=3$. Let $\left\{Z_{1}, Z_{2}, Z_{3}\right\}$ be an orthonormal basis of $\mathfrak{z}$ and $X \in \mathfrak{v}$ a unit vector satisfying

$$
J_{1} J_{2} J_{3} X=X \text {. }
$$

To get compact formulæ, it is useful to introduce the tensor $\epsilon_{i j k}$ which is by definition invariant under circular permutations of the indexes and satisfies $\epsilon_{123}=-\epsilon_{213}=1$, $\epsilon_{i i j}=0, i, j \in\{1,2,3\}$. The symbol $\epsilon_{i j k}$ satisfies the identities

$$
\sum_{m=1}^{3} \epsilon_{i j m} \epsilon_{m k l}=\delta_{i k} \delta_{j l}-\delta_{i l} \delta_{j k} . \quad \text { and } \quad \sum_{l, m=1}^{3} \epsilon_{i l m} \epsilon_{l m j}=2 \delta_{i j} .
$$

Now, using the first of the (A.2.2)'s, it is easy to see that

$$
J_{i} X=-\frac{1}{2} \sum_{m=1}^{3} \epsilon_{i m n} J_{m} J_{n} X \quad \text { and } \quad J_{i} J_{k} X=-\delta_{i k} X-\sum_{m=1}^{3} \epsilon_{i k m} J_{m} X,
$$

from which it follows

$$
J_{i} J_{k} J_{l} X=\delta_{i l} J_{k} X-\delta_{k i} J_{l} X-\delta_{k l} J_{i} X+\epsilon_{i k l} X .
$$

From (A.2.3) one obtains

$$
\left[X, J_{l} J_{k} X\right]=-\sum_{i=1}^{3} \epsilon_{i l k} Z_{i} \quad l, k \in\{1,2,3\} .
$$

Moreover, since

$$
\left\langle Z_{l},\left[J_{i} X, J_{k} X\right]\right\rangle=\left\langle J_{l} J_{i} X, J_{k} X\right\rangle=-\left\langle J_{k} J_{l} J_{i} X, X\right\rangle=-\epsilon_{k l i},
$$

it follows that

$$
\left[J_{i} X, J_{k} X\right]=-\sum_{l=1}^{3} \epsilon_{i k l} Z_{l} \quad l, k \in\{1,2,3\},
$$

which, according to Lemma 2.2 , is equal to $\left[X, J_{i} J_{k} X\right]$. 


\section{References}

[C1] P. Ciatti, A Clifford algebra approach to simple Lie algebras of real rank two, I: The $A_{2}$ case, J. Lie Theory 10 (2000), 53-80.

[C2] P. Ciatti, A new proof of the $J^{2}$-condition for real rank one simple Lie algebras and their classification, (to appear in Proc. AMS).

[CC] P. Ciatti and M. Cowling, The automorphism group of an Iwasawa nilpotent algebra, (preprint).

[CDKR1] M. Cowling, A. Dooley, Korányi and F. Ricci, H-type groups and Iwasawa decomposition, Adv. Math. 87 (1997), 1-4.

[CDKR2] M. Cowling, A. Dooley, Korányi and F. Ricci, An approach to symmetric spaces of rank one via groups of Heisenberg type, J. Geom. Anal. 8 (1998), 199-237.

[K] A. Kaplan, Fundamental solutions for a class of hypoelliptic PDE generated by compositions of quadratic forms, Trans. Amer. Math. Soc. 258 (1980), 147-153.

[Ko] A. Korányi, Geometric properties of Heisenberg type groups, Adv. Math. 56 (1986), $28-38$.

[P] I. Porteous, Clifford Algebras and the Classical Groups, Cambridge University Press, Cambridge, 1995.

[R] C. Riehm, The automorphism group of a composition of quadratic forms, Trans. Amer. Math. Soc. 269 (1982), 403-414.

[S] L. Saal, The automorphism group of a Lie algebra of Heisenberg type, Rend. Sem. Mat. Univ. Pol. Torino 54 (1996), 101-113. 